\title{
Einflussfaktoren und Folgen des Ausfalls gesetzlicher Ruhepausen bei Pflegekräften in Deutschland
}

\author{
Andrea Lohmann-Haislah ${ }^{1}$ Johannes Wendsche ${ }^{2}$. Anika Schulz • Ina Schöllgen ${ }^{3}$ Luis Carlos Escobar Pinzon ${ }^{4}$
}

Online publiziert: 4. November 2019

(c) Der/die Autor(en) 2019, korrigierte Publikation 2020

\section{Zusammenfassung}

Der Pflegeberuf ist mit hoher körperlicher und psychischer Belastung verbunden. Infolgedessen finden sich in der Krankenund Altenpflege erhöhte Risiken für körperliche und psychische Beschwerden. Dabei dürfte sich die Belastungssituation in den letzten Jahren aufgrund struktureller und organisationaler Veränderungen im Pflegebereich, gestiegenem Pflegeaufwand und Problemen mit einer ausreichenden Fachkräftesicherung verstärkt haben. Insofern ist es wichtig, solche Arbeitsmerkmale zu stärken, die beeinträchtigende Beanspruchungsfolgen zu mindern vermögen und damit eine gesunde und sichere Arbeit ermöglichen. Arbeitspausen stellen eine solche Arbeitsressource dar. In Deutschland sind Mindestpausenzeiten gesetzlich verpflichtend. Verschiedene Studien zeigten allerdings, dass Pausen bei Pflegetätigkeiten überdurchschnittlich häufig ausfallen, verkürzt werden oder zeitlich nicht wie geplant genommen werden können. Über Treiber und Folgen des Ausfalls von gesetzlichen Ruhepausen ist allerdings wenig bekannt. Dies motivierte zu der nachfolgenden Untersuchung. Grundlage der Auswertungen waren für Deutschland repräsentative Befragungsdaten von Erwerbstätigen, darunter Beschäftigte aus dem Bereich Kranken- und Altenpflege $(N=1003$; Krankenpflege $n=685$, Altenpflege $n=318)$, die im Rahmen der im Querschnitt angelegten 6. Welle der BIBB/BAuA-Erwerbstätigenbefragung 2012 gewonnen wurden. Es zeigte sich, dass Pausen signifikant häufiger im Alten- und Krankenpflegebereich ausfallen als bei sonstigen Beschäftigten. Als Hauptgründe für den Pausenausfall werden sowohl in der Kranken- als auch in der Altenpflege hohe Arbeitsmengen angegeben. Weitere Analysen zeigen, dass fehlende Informationen, Merkmale hoher Arbeitsintensität, Schichtarbeit, das Innehaben einer Führungsposition, mangelnde Selbstbestimmung beim Pausenzeitpunkt und Wochenendarbeit mit erhöhtem Pausenausfall einhergehen. Zudem lassen Beschäftigte mit einem unbefristeten Vertrag häufiger die Pause ausfallen, als die mit einem befristeten Vertrag. Bei Betrachtung psychosomatischer Beschwerden zeigt sich, dass die Beschäftigten in der Pflege mit Pausenausfall häufiger psychosomatische Beschwerden angeben als die ohne Pausenausfall, ebensolches gilt für die Muskel-Skelett-Beschwerden. Es wird aber auch deutlich, dass Pausen selbst unter hohen Arbeitsanforderungen diese negativen Beanspruchungsfolgen reduzieren können.

Praktische Relevanz: Zur Sicherung der Versorgung im Gesundheitswesen sowie der Gesundheit und Beschäftigungsfähigkeit der dort arbeitenden Pflegekräfte sollte dem Ausfall von gesetzlichen Ruhepausen in Zukunft besser vorgebeugt werden.

Schlüsselwörter Anforderungen $\cdot$ Erholung $\cdot$ Pflege $\cdot$ Pausen $\cdot$ Ressourcen

Dipl.-Psych. Andrea Lohmann-Haislah

lohmann-haislah.andrea@baua.bund.de

Dr. Johannes Wendsche

wendsche.johannes@baua.bund.de

Anika Schulz, M.Sc.

schulz.anika2@baua.bund.de

Dr. Ina Schöllgen

schoellgen.ina@baua.bund.de

PD Dr. Luis Carlos Escobar Pinzon

EscobarPinzon.LuisCarlos@baua.bund.de
1 Gruppe 3.5 Psychische Belastungen, Bundesanstalt für Arbeitsschutz und Arbeitsmedizin, Nöldnerstraße 40-42, 10317 Berlin, Deutschland

2 Gruppe 3.6 Betriebliche Intervention und Transfer, Bundesanstalt für Arbeitsschutz und Arbeitsmedizin, Fabricestr. 8, 01099 Dresden, Deutschland

3 Gruppe 3.4 Mentale Gesundheit und Kognitive Leistungsfähigkeit, Bundesanstalt für Arbeitsschutz und Arbeitsmedizin, Nöldnerstraße 40-42, 10317 Berlin, Deutschland

4 Fachbereich 3 Arbeit und Gesundheit, Bundesanstalt für Arbeitsschutz und Arbeitsmedizin, Nöldnerstraße 40-42, 10317 Berlin, Deutschland 


\title{
Determinants and outcomes of skipping mandatory rest breaks in German nurses
}

\begin{abstract}
The nursing profession is subject to high physical and psychological stressors. As a result, these occupational groups (nurses in hospitals and elderly care) are exposed to increased risks of impairing physical and psychological complaints. This stressful situation was likely to become more dramatic in recent years as a result of structural and organizational changes in the nursing sector, increased nursing expenditure and problems with hiring sufficiently skilled staff. In this respect, it is important to strengthen work characteristics that are able to reduce the adverse effects of work stressors and, thus, enable healthy and safe work. Work breaks are such a job resource. In Germany, minimum break times are legally mandatory. However, some studies have shown that breaks in nursing are often skipped, shortened in time or cannot be taken as planned. However, little is known about the drivers and consequences of the absence of mandatory breaks, which motivated us to conduct the following study. The analyses are based on representative survey data of German employees, including nurses $(N=1003)$ in hospitals $(n=685)$ and elderly care $(n=318)$. These cross-sectional data were collected in the sixth wave of the BIBB/BAuA employee survey 2012. We found that skipped breaks were significantly more frequent for nurses working in elderly care and hospitals than in other professions. The main reason for skipping breaks was high workload for both, nurses working in hospitals and in elderly care. Further analyses showed that a lack of information, attributes of high work intensity, shift work, holding a managerial position, low break autonomy and weekend work are associated with increased risks of skipping mandatory breaks. In addition, employees with a permanent job contract miss their breaks more often than those with a fixed-term contract. With regard to psychosomatic complaints our results showed that nurses skipping breaks report more psychosomatic complaints than those without, for musculoskeletal complaints as well. However, we found that breaks can reduce these negative strain outcomes even in case of high work demands.

Practical relevance: In order to safeguard healthcare provision and the health and employability of the nursing staff working there, preventing the skipping of mandatory breaks must be improved upon in the future.
\end{abstract}

Keywords Demands $\cdot$ Nursing staff $\cdot$ Recovery $\cdot$ Resources $\cdot$ Rest break $\cdot$ Work break

\section{Einleitung}

Der Anteil an Bedürftigen für professionelle Pflege und damit auch der Bedarf an Pflegekräften nehmen zu. So waren Ende 2015 knapp 2,9 Mio. Menschen im Sinne des Pflegeversicherungsgesetzes (SGB XI) pflegebedürftig. Das sind rund 74\% mehr als im Jahre 1999. In ambulanten Pflegediensten und stationären Pflegeeinrichtungen waren 1999 knapp 1,1 Mio. Personen nach SGB XI beschäftigt, bis 2015 war hier ein Anstieg um rund $77 \%$ zu verzeichnen (Statistisches Bundesamt 2017).

Trotz dieser Zuwächse ist bereits seit einiger Zeit ein steigender Fachkräftemangel in allen Pflegeberufen zu konstatieren. Laut der Fachkräfteengpassanalyse der Bundesagentur für Arbeit sind Stellenangebote für examinierte Altenpflegefachkräfte und -spezialisten im Bundesdurchschnitt 171 Tage unbesetzt. Auf 100 gemeldete Stellen (außerhalb der Zeitarbeit) kommen rechnerisch lediglich 29 Arbeitslose (Bundesagentur für Arbeit 2018). Unter der Annahme, dass die Pflegefallwahrscheinlichkeiten und der Personalbedarf für Pflegebedürftige unverändert bleiben (,Status-quo-Szenario“), könnte der Bedarf an Pflegevollkräften (sowohl Fachkräfte als auch Pflegehelfer) bis 2025 um rund $27 \%$ gegenüber 2005 ansteigen. Dies könnte bei konstanter Fortschreibung der Beschäftigungsstruktur zu einer Lücke von ausgebildeten Pflegekräften von rund 200.000 Pflegekräften im Jahre 2025 führen (Afentakis und Maier 2010). Für die ambulante und stationäre Pflege insgesamt geht die Vereinigung der Bayerischen Wirtschaft e.V. (2012) sogar von einer Versorgungslücke von bis zu 737.000 im Jahre 2030 (entspricht ca. 520.000 Vollzeitäquivalenten) aus, was sich ungefähr mit dem Themenreport Pflege 2030 der Bertelsmann Stiftung (Rothgang et al. 2012) deckt, der von einer Lücke von rund 490.000 Vollzeitäquivalenten in 2030 spricht. Vor dem Hintergrund vielfach diskutierter schlechter Arbeitsbedingungen in den Pflegeberufen mit ihrer hohen körperlichen und psychischen Belastung bergen die vergleichsweise hohen Zahlen bei den Arbeitsunfähigkeitsdaten und den Frühverrentungen, die relativ geringe Verweildauer im Beruf und die hohe Fluktuationsneigung bzw. die hohe tatsächliche Fluktuation nicht zu vernachlässigende zusätzliche Risiken hinsichtlich der zukünftigen Versorgung mit ausreichendem Pflegepersonal in Deutschland.

Um diesen Risiken zu begegnen, werden unter anderem eine bessere Bezahlung, ein besserer Personalschlüssel und vereinfachte Zugänge zur Berufsausbildung diskutiert (Hasselhorn et al. 2005). Doch sollten darüber hinaus auch die Arbeitsbedingungen in Gesundheitsberufen in den Blick genommen werden und deshalb bestehende körperliche und psychische Anforderungen des Pflegeberufes optimiert werden (Glaser und Höge 2005). Auf der anderen Seite gilt 
es jedoch auch solche Arbeitsressourcen zu fördern, die negative Folgen von Belastung zu minimieren vermögen. Dazu gehören nicht nur die klassischen Gestaltungsmerkmale wie Handlungs- und Entscheidungsspielräume sowie soziale Unterstützung, sondern auch genügend Erholungsmöglichkeiten. Neben guter Schichtplangestaltung und ausreichenden Ruhezeiten, zählen dazu auch Pausen während der Arbeitszeit. Doch vielfach werden in diesen Berufen nicht einmal die gesetzlichen Mindestpausenzeiten eingehalten. Erst vereinzelt wehren sich Beschäftigte dagegen, wie beispielsweise das Pflegepersonal in einer Berliner Klinik, das aufgrund ausfallender Pausen vor dem Arbeitsgericht klagte, was ein Bußgeld von $88.000 €$ für den Klinikbetreiber nach sich zog (Gehrke 2017 in Berliner Zeitung, 22./23. April 2017).

Wie es mit dem Pausenausfall in der Pflege im Vergleich $\mathrm{zu}$ anderen Berufen nun genau bestellt ist, welche Arbeitsbedingungen zu Pausenausfall beitragen und welche $\mathrm{Zu}$ sammenhänge mit gesundheitlichen Beschwerden bestehen, ist der wesentliche Inhalt dieses Beitrags. Zuvor werden die Arbeitsbedingungen in den Pflegeberufen genauer beleuchtet und theoretische Hintergründe für den Pausenausfall und dessen Folgen dargestellt.

\section{Theoretischer Hintergrund}

In einer Weiterentwicklung arbeitswissenschaftlicher Belastungs-Beanspruchungs-Modelle sowie arbeitspsychologischer Job-Design-Theorien haben Demerouti und Kollegen das Arbeitsanforderungen-Arbeitsressourcen-Modell vorgeschlagen, um unterschiedliche Wirkungen von Arbeit auf den Beschäftigten genauer erklären zu können (im Überblick Demerouti und Nachreiner 2018). Das Modell geht davon aus, dass zwei grundsätzliche Gruppen von Arbeitsbedingungsfaktoren existieren. Unter Arbeitsanforderungen werden physische, psychische, soziale und organisatorische Merkmale der Arbeit verstanden, die $\mathrm{zu}$ Beanspruchungen beim Beschäftigten führen und in Folge mit physiologischen und/oder psychischen Kosten einhergehen (Beanspruchungsfolgen sensu DIN EN ISO 10075 Teil 1 2018). Im Modell wird angenommen, dass Anforderungen vor allem mit kurz- und langfristigen beeinträchtigenden Beanspruchungsfolgen (z.B. Ermüdung, Erschöpfung) positiv zusammenhängen. Im Gegensatz dazu sind Arbeitsressourcen physische, psychische, soziale und organisatorische Merkmale der Arbeit, die der Erreichung von Arbeitszielen dienen, Arbeitsanforderungen und begleitende beeinträchtigende Beanspruchungsfolgen reduzieren und durch förderliche Lern- und Weiterentwicklungswirkungen charakterisiert sind. Sie sind entsprechend der Modellannahmen mit positiven motivationalen Wirkungen verbunden und vermögen es - auch entsprechend der Definition - negative Beanspruchungsfolgen bei Arbeitsbedingungen mit hohen Anforderungen abzumildern. Aber wie sieht die Belastungsstruktur (Arbeitsanforderungen und Arbeitsressourcen) bei Pflegetätigkeiten aus und welche Beanspruchungswirkungen werden im Vergleich zu anderen Berufsgruppen berichtet?

\subsection{Arbeit in der Pflege}

In einer aktuellen repräsentativen Studie der Hans-Böckler-Stiftung, bei der ein Ländervergleich der ambulanten und stationären Pflege in Deutschland, Schweden und Japan durchgeführt wurde, wird festgestellt, dass in den drei Ländern Arbeit in der Pflege sowohl mit hoher körperlicher als auch mit hoher psychischer Belastung und Beanspruchung einhergeht (Theobald 2018). Der Studie zufolge geben in Deutschland und Schweden 40 bis $50 \%$ aller Befragten im ambulanten Sektor sowie 60 bis $70 \%$ im stationären Sektor an, täglich schwere Dinge oder Personen bewegen zu müssen. In Japan sind es in der ambulanten Pflege, die dort vor allem Haushaltstätigkeiten umfasst, $18 \%$ sowie $81 \%$ in Pflegeheimen. Nach einem Arbeitstag in der ambulanten Pflege sind $46 \%$ der Beschäftigten in Deutschland sowie etwa $60 \%$ in Schweden und Japan fast immer körperlich erschöpft. Von den Beschäftigten in Pflegeheimen berichteten in Deutschland und Schweden $70 \%$ über körperliche Erschöpfung, in Japan mehr als $80 \%$ (Theobald 2018). Die Pflege alter Menschen wird in allen drei Ländern als belastend empfunden. Darüber hinaus berichten $54 \%$ der ambulanten Pflegekräfte in Deutschland, 35\% in Japan und $37 \%$ in Schweden von regelmäßigem Zeitdruck. Wöchentliche Überstunden fallen bei $52 \%$ der Deutschen, $28 \%$ der Japaner und $13 \%$ der Schweden an (Theobald 2018). Die Ergebnisse für Deutschland im Rahmen des Ländervergleichs korrespondieren mit den Auswertungen der BIBB-BAuA-Erwerbstätigenbefragung. Ihr zufolge gibt mehr als die Hälfte aller Befragten aus Gesundheits- und Krankenpflegeberufen an, schnell arbeiten zu müssen. Mehr als drei Viertel berichten von der häufigen Notwendigkeit, verschiedene Arbeiten gleichzeitig erledigen zu müssen. Hinzu kommen weitere psychisch belastende Faktoren wie Schicht- und Nachtarbeit (Bundesanstalt für Arbeitsschutz und Arbeitsmedizin 2014). Des Weiteren sind Pflegekräfte, gerade in speziellen Sektoren wie der Palliativversorgung, durch die Betreuung von Angehörigen sowie den Zeitaufwand zum Informationsaustausch mit anderen (Fach-)Kräften ebenfalls belastet (Diehl et al. 2018).

Die Datenanalysen des aktuellen BKK-Gesundheitsatlas deuten darauf hin, dass viele Pflegekräfte einen schlechten Gesundheitszustand und längere Fehlzeiten haben. Weibliche Beschäftigte in Pflegeheimen, die den überwiegenden Teil der Beschäftigten bilden, haben beispielsweise aufgrund psychischer Beeinträchtigungen doppelt so vie- 
le Krankheitstage wie der Durchschnitt aller Beschäftigten: 4,6 Krankentage in Pflegeheimen gegenüber 2,3 Tage in anderen Branchen. Aufgrund von Muskel- und Skeletterkrankungen fallen Altenpflegerinnen fast doppelt so lange aus wie sonstige weibliche Beschäftigte $(7,0$ versus 3,7 Krankentage). Innerhalb der Gruppe der medizinischen Gesundheitsberufe sind es die Gesundheits- und Krankenpfleger, bei denen mit durchschnittlich 19,3 AU-Tagen je beschäftigtem Mitglied der BKK die meisten Fehlzeiten zu finden sind. Insbesondere die weiblichen Beschäftigten in dieser Berufsgruppe liegen mit knapp 20 AU-Tagen deutlich vor ihren männlichen Kollegen mit 16,7 AU-Tagen. Im Vergleich zu allen anderen Beschäftigten, die im Schnitt 16 Tage krank waren, haben Pflegekräfte in Pflegeheimen mit rund 24 Tagen deutlich längere Ausfallzeiten (Kliner et al. 2017).

Insgesamt ist festzuhalten, dass im Vergleich zu anderen Berufsfeldern und -gruppen in der Pflege sehr hohe körperliche und gleichzeitig psychische Arbeitsanforderungen vorliegen und dass die Prävalenzraten sowohl für körperliche als auch psychische Beschwerden erhöht sind. Es kann vermutet werden, dass an letzteren auch eine mangelhafte Gestaltung potentiell förderlicher Arbeitsressourcen beteiligt ist. Eine dieser Ressourcen ist die Gestaltung von Arbeitspausen. Im nachfolgenden Abschnitt beschreiben wir gesetzliche Regeln zur Pausenorganisation in Deutschland und diskutieren, welche Erkenntnisse zu ihren EinflussgröBen und zu ihren Wirkungen existieren.

\subsection{Einflussfaktoren und Wirkungen der Pausenorganisation in Pflegeberufen}

\subsubsection{Gesetzliche Regeln}

In Europa sind grundlegende Anforderungen zur Gestaltung der Arbeitszeit von abhängig Beschäftigten in der EU-Arbeitszeitrichtlinie (2003/88/EG) enthalten. Die Umsetzung dieser Regeln im deutschen Arbeitszeitgesetz (ArbZG §4) sieht vor, dass Beschäftigte mit einer täglichen Mindestarbeitszeit von sechs Stunden einen Anspruch auf vor Arbeitsbeginn geplante und, wenn nicht anders geregelt, unbezahlte Ruhepausen von mindestens 30 min (ab neun Stunden täglicher Arbeitszeit $45 \mathrm{~min}$ ) haben. Eine Aufteilung der Gesamtdauer dieser Ruhepausen in mindestens 15-minütige Abschnitte ist möglich. Bisher ist allerdings wenig darüber bekannt, wie häufig solche Pausen bei Pflegekräften in verschiedenen Arbeitsbereichen (Kranken- vs. Altenpflege) ausfallen und ob dabei substanzielle Unterschiede $\mathrm{zu}$ anderen Berufsgruppen vorliegen. Wir fragen daher als erstes:
Fragestellung 1 Wie häufig fallen gesetzliche Ruhepausen bei Kranken- und Altenpflegekräften im Vergleich zu anderen Berufsgruppen aus?

Im nächsten Schritt gilt es nach den Gründen für den Ausfall von Pausen zu fragen bzw. nach den Einflussgrößen der Einhaltung von Arbeitspausen zu suchen - im Besonderen bei Beschäftigten im Pflegebereich.

\subsubsection{Einflussgrößen auf Pausenorganisation}

In einer Übersichtsarbeit haben Wendsche et al. (2017) den internationalen Forschungstand zur Pausenorganisation im Pflegebereich zusammengefasst. Sie fanden, dass über verschiedene Länder hinweg ein Großteil der untersuchten Pflegekräfte ungünstige Pausenregime (Ausfall, Verkürzung und/oder Verlagerung von Pausen) berichtet. Dies legt die Vermutung nahe, dass es weniger länderspezifische Regeln oder individuelle Faktoren sind, die das Pausenverhalten beeinflussen, sondern generelle Arbeits- und Organisationsbedingungen in diesem Beruf. Im Einklang mit dieser Annahme fanden sich in den Studien keine eindeutigen Belege, dass demografische Faktoren wie Alter und Geschlecht eine substanzielle Rolle für die Pauseneinhaltung spielen. Vielmehr zeigte der Großteil an Studien, dass hohe zeitliche und mengenmäßige Anforderungen aus der Arbeit (z. B. Zeitdruck, Personalmangel, geringe Fachkraftquote, Arbeit auf Intensivpflegestationen) mit einer Verkürzung bzw. dem Ausfall von Arbeitspausen assoziiert sind. Für die Beziehung zwischen Arbeitszeitmerkmalen (Dauer, Schichtarbeit, Rotationssystem) und der Einhaltung von Arbeitspausen fanden sich bisher inkonsistente Befundmuster, die allerdings auch dadurch erklärt werden können, dass solche Merkmale selektiv und weder in Kombination noch im Zusammenspiel mit anderen Arbeitsmerkmalen untersucht wurden. Es gab Hinweise, dass sowohl Arbeitsressourcen (z.B. hohe soziale Unterstützung durch Kollegen, hohe Kontrolle über die Arbeitszeitgestaltung, klare und faire Pausenregeln) als auch räumliche Ressourcen (z. B. separate und erholungsförderliche gestaltete Pausenbereiche) positiv mit der Pausennahme assoziiert sind. Zu klären ist, ob die Pflegekräfte selbst diese in Zusammenhangsanalysen ermittelten Faktoren als Ursachen des Pausenausfalls wahrnehmen. Deshalb fragen wir in unserer Untersuchung zunächst nach individuellen Attributionen zu den Ursachen des Ausfalls gesetzlicher Ruhepausen:

Fragestellung 2 Welche Gründe für den Ausfall gesetzlicher Ruhepausen nennen Kranken- und Altenpflegekräfte im Vergleich zu anderen Berufsgruppen?

Nun gilt es jedoch auch zu fragen, wie sich die vorher geschilderten Befunde theoretisch erklären lassen. Zwar gibt es kein Gesamtmodell, aber aus unserer Sicht sind hier mehrere Ansätze hilfreich. Beispielsweise besagt die Rol- 
lentheorie von Katz und Kahn (1978), dass Beschäftigte innerhalb einer Organisation bestimmte Rollen entsprechend ihrer Position und ihrer Tätigkeiten übernehmen. Diese Rollen sind mit übernommenen und entwickelten Erwartungen an das Arbeitsverhalten verbunden. So hatten beispielsweise Lohmann-Haislah und Bodnar (2014) festgestellt, dass Führungskräfte häufiger als Mitarbeiter ohne Führungsverantwortung ihre Ruhepausen ausfallen lassen. Es kann vermutet werden, dass dies nicht nur auf andere Arbeitsbedingungen zurückzuführen ist, sondern möglicherweise auch auf die aus der Führungsrolle übernommene, erwartete hohe Anstrengungsbereitschaft. Der Verzicht auf Pausen würde demnach Leistungswillen symbolisieren und vorbildhaft für das Arbeitsverhalten der Gruppenmitglieder wirken. Es wäre zu vermuten, dass sich ähnliche Befunde auch im Pflegebereich zeigen. Daraus folgt unsere Annahme:

Hypothese 1 In Pflegeberufen lassen Beschäftigte mit Führungsverantwortung häufiger gesetzliche Ruhepausen ausfallen als solche ohne Führungsverantwortung.

Ähnliche Wirkungen dürfte entsprechend der Rollentheorie und der sozialen Austauschtheorie (Blau 1964; Gouldner 1960) auch die Befristung des Arbeitsverhältnisses auf das Pausenverhalten haben. So kann vermutet werden, dass befristet Beschäftigte im Vergleich zu unbefristeten eher Pausen ausfallen lassen, in der Hoffnung, dass ihr Verhalten als Kennzeichen hoher Arbeitsmotivation erkannt wird und schließlich - im Austausch - in eine Vertragsentfristung und einer dauerhaften Aufnahme in die Arbeitsgruppe mündet.

Allerdings wären bezüglich der Befristung auch gegenläufige Zusammenhänge denkbar. Denn insbesondere längere Ruhepausen werden oft zusammen mit Kollegen verbracht (Wendsche und Lohmann-Haislah 2018). Dabei ist der arbeitsbezogene Austausch in den Pausen nicht nur eine Bewältigungsstrategie im Umgang mit den Arbeitsanforderungen und geht mit weniger Erschöpfungssymptomen einher (Ishig und Hacker 1999), sondern diese Art soziale Pause hat auch eine identitätsstiftende Funktion und fördert den Gruppenzusammenhalt und die organisationale Bindung (Stefancyk 2009; Wendsche et al. 2014). Zudem sagt die soziale Identitätstheorie (Tajfel und Turner 1986) vorher, dass Menschen im Streben nach einer positiven Selbsteinschätzung und sozialer Identität versuchen, von einer statusniedrigeren (befristeter Arbeitsvertrag) in eine statushöhere (entfristeter Arbeitsvertrag) Gruppe aufzusteigen (Wilkin et al. 2018). Dies gelingt u. a. durch das Herstellen von Kontakt- und Austauschmöglichkeiten mit der anderen Gruppe, z. B. in Gruppenpausen. Insofern könnte also auch vermutet werden, dass befristet Beschäftigte die Pause seltener ausfallen lassen als unbefristete. Darüber hinaus könnte eine geringere Prävalenz des Ausfalls von Ruhepausen auch durch andere Faktoren bedingt sein. So fanden Salo- niemi et al. (2004) in einer großen finnischen Studie günstigere psychosoziale Arbeitsbedingungen (geringere Anforderungen, mehr Kontrolle, mehr Unterstützung) bei befristeten im Vergleich zu unbefristeten Beschäftigten, also solchen Arbeitsbedingungen, die das Risiko für das Auslassen von Arbeitspausen reduzieren (Wendsche et al. 2017). Aufgrund dieser theoretisch abgeleiteten unterschiedlichen Wirkzusammenhänge fragen wir deshalb hier:

Fragestellung 3 Unterscheidet sich die Häufigkeit des Ausfalls gesetzlicher Ruhepausen bei vertraglich befristeten im Vergleich zu unbefristeten Pflegekräften?

Einen weiteren Erklärungsansatz zur Begründung des Ausfalls von Pausen liefert das Rahmenmodell der Arbeitsintensität (Rau und Göllner 2018). Demnach versuchen Beschäftigte die geforderte Arbeitsmenge, das kognitive Regulationsniveau zur Aufgabenbewältigung und die verfügbare Zeit miteinander so in Einklang zu bringen, dass der übernommene Arbeitsauftrag bei erträglicher Arbeitsintensität erfüllt werden kann. Ergeben sich nun Konflikte zwischen diesen Regulationsgrößen, z. B. aufgrund von Personalmangel, hohen Arbeitsmengen (z.B. Zeitdruck), Leistungsdruck, enger Zeitbindung, hohen kognitiven und/oder emotionalen Arbeitsanforderungen oder Hindernissen bei der Auftragsbewältigung (z. B. Arbeitsunterbrechungen, Informationsmangel), so besteht eine Lösungsmöglichkeit darin, die geforderte Auftragserfüllung durch zeitsparende Bewältigungsstrategien zu sichern (Krause et al. 2017): das Ausfallenlassen von Pausen. Oftmals tritt dieses Verhalten auch gleichzeitig mit anderen, arbeitsextensivierenden Strategien auf, wie beispielsweise Mehrarbeit und Überstunden (Schulz-Dadaczynski 2017). So fanden Tanner et al. (2017) in einer Untersuchung mit Klinikärzten eine signifikante negative Korrelation zwischen der Anzahl an Überstunden und der Einhaltung von Pausen $(r=-0,33)$.

Darüber hinaus können bestimmte Arbeitsanforderungen nach dem Challenge-Hindrance-Stressor-Modell (Cavanaugh et al. 2000; LePine et al. 2005; Podsakoff et al. 2007) auch eine starke motivationale Kraft entfalten, weil sie als Herausforderung wahrgenommen werden und damit individuelle Wachstums- und Leistungsmotive befriedigen. $\mathrm{Zu}$ diesen Arbeitsmerkmalen zählen beispielsweise die geforderte Arbeitsmenge, Zeitdruck bei der Arbeit, hohe Verantwortung und hohe Aufgabenkomplexität. Aufgrund ihrer starken motivationalen Kraft können diese Arbeitsbedingungen zu Flow-Erlebnissen führen. Diese sind durch $\mathrm{Spa}$ an der Tätigkeit, einem Aufgehen in diesen starken positiven Emotionen sowie einem hohem Belohnungserleben und einem reduzierten Zeitgefühl gekennzeichnet (Nakamura und Csikszentmihalyi 2014). Es wird deshalb kein Anlass für die erholungswirksame Unterbrechung der Arbeit gesehen, z. B. weil die Zielerreichung im Vordergrund des Handelns steht oder (physiologische) Beanspruchungs- 
signale nicht wahrgenommen werden (Keller et al. 2011). Daraus folgt:

Hypothese 2 In Pflegeberufen berichten Mitarbeiter mit höheren psychischen Arbeitsanforderungen häufiger den Ausfall gesetzlicher Ruhepausen als mit niedrigeren.

Als zentrale Kriterien werden hier folgende Arbeitsmerkmale verstanden: (a) emotionale Anforderungen, (b) quantitative Anforderungen (Termin- und Leistungsdruck, Zwang zum schnellen Arbeiten, Arbeiten bis zur Grenze der Leistungsfähigkeit) und (c) hohe kognitive Anforderungen (fehlende Informationen zur Aufgabenbewältigung, Arbeitsunterbrechungen, Multitaskinganforderungen).

Hinsichtlich des Einflusses von Arbeitszeitmerkmalen auf den Ausfall von Ruhepausen ergeben sich aus den vorhergehenden theoretischen Erläuterungen außerdem die folgenden zwei Annahmen:

Hypothese 3 In Pflegeberufen berichten Mitarbeiter mit einer längeren Arbeitszeit (inkl. Mehrarbeits- und Überstunden) häufiger den Ausfall gesetzlicher Ruhepausen als mit kürzeren.

Hypothese 4 In Pflegeberufen berichten Mitarbeiter mit geringeren zeitlichen Entscheidungsspielräumen bei der Pauseneinnahme häufiger den Ausfall gesetzlicher Ruhepausen als mit höheren.

Die Befunde zur Wirkung anderer Arbeitszeitmerkmale waren bisher inkonsistent (Wendsche et al. 2017) und sollen hier explorativ untersucht werden.

Fragestellung 4 Welche Zusammenhänge bestehen zwischen (a) Schichtarbeit sowie (b) Wochenendarbeit und dem Ausfall gesetzlicher Ruhepausen bei Pflegekräften?

\subsubsection{Wirkungen von Arbeitspausen}

In der Überblicksarbeit von Wendsche et al. (2017) zeigte sich in der Mehrheit der Studien, dass Pflegekräfte mit regelmäßigeren und längeren Pausen weniger körperliche Beschwerden und ein höheres Wohlbefinden berichten. Es fanden sich auch Befunde, dass Arbeitspausen mit den Einstellungen zur Arbeit und zur Arbeitsleistung zusammenhängen, beispielsweise als Erleben von mehr sozialer Unterstützung, einer höheren Arbeitsmotivation, einer höheren Arbeitszufriedenheit, einer geringeren Fluktuationsneigung und Fluktuationsrate sowie weniger Pflegefehlern. Solche positiven Wirkungen von Arbeitspausen sowohl auf das körperliche und psychische Wohlbefinden, die Arbeitsleistung sowie die Arbeitssicherheit konnten inzwischen in Metaanalysen für verschiedene Berufsgruppen belegt werden (Fischer et al. 2017; Wendsche et al. 2016; Wendsche und Lohmann-Haislah 2018). Theoretisch erklären lassen sich die förderlichen Wirkungen von Arbeitspausen zur Vor-

beugung beeinträchtigender körperlicher und psychischer Beanspruchungsfolgen durch die arbeitswissenschaftlichen Erkenntnisse zu Zusammenhängen zwischen Belastungsdauer, Erholung und Beanspruchungsfolgen (Graf et al. 1970; Meijman und Mulder 1998). Demnach beugen Arbeitspausen durch eine Begrenzung der Belastungsdauer kritischen beeinträchtigenden Beanspruchungszuständen vor und reduzieren während der Erholungsphase das Niveau an negativen Beanspruchungsfolgen.

Wir nehmen deshalb Folgendes an:

Hypothese 5 In Pflegeberufen erleben Beschäftigte, die den regelmäßigen Ausfall von gesetzlichen Ruhepausen berichten, mehr körperliche Beschwerden als solche, die den Ausfall von gesetzlichen Ruhepausen nicht berichten.

Hypothese 6 In Pflegeberufen erleben Beschäftigte, die den regelmäßigen Ausfall von gesetzlichen Ruhepausen berichten, mehr psychosomatische Beschwerden als solche, die den Ausfall von gesetzlichen Ruhepausen nicht berichten.

\section{Methoden}

\subsection{Stichprobenbeschreibung und Untersuchungsdurchführung}

Unsere Analysen beruhen auf den Daten der 6. Welle der BIBB/BAuA-Erwerbstätigenbefragung 2012. Dabei wurde eine repräsentative Stichprobe von rund 20.036 erwerbstätigen Personen in Deutschland (ab 15 Jahren, bezahlte Tätigkeit von mindestens $10 \mathrm{~h} /$ Woche Arbeitszeit, ausreichende Deutschkenntnisse) durch TNS Infratest Sozialforschung in einer telefonischen, computerunterstützten Befragung (CATI) zu ihrer Qualifikation, ihren Arbeitsbedingungen und ihrem körperlichen und psychischen Befinden befragt (Wittig et al. 2013). In der Auswertungsstichprobe von abhängig Beschäftigten $(n=17.562)$ nutzen wir nachfolgend nur diejenigen, die Angaben zum Pausenausfall gemacht hatten $(n=17.178)$. Zur Stratifizierung unserer Analysen wurden aus der Klassifikation der Berufe 2010 (KldB 2010; Bundesagentur für Arbeit 2011) anhand der 3stelligen Berufscodes die zwei Gruppen Krankenpflegende (KldB: 813 Gesundheits- und Krankenpflege, Rettungsdienst und Geburtshilfe) und Altenpflegende (KldB: 821 - Altenpflege) gebildet. Alle anderen abhängig Beschäftigten fielen unter „sonstige“ ( $n=16.175)$. Eine deskriptive Beschreibung der Substichproben kann der Tab. Tab. 1 entnommen werden. 


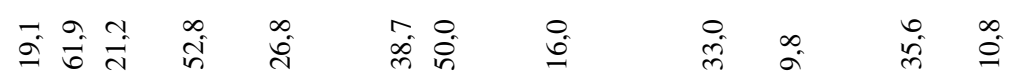

$\frac{7}{2}$

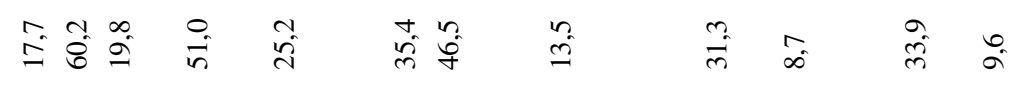

तेंूू̆

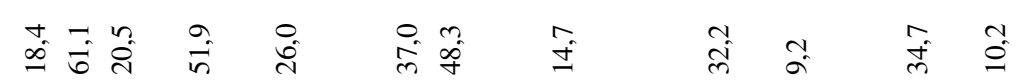

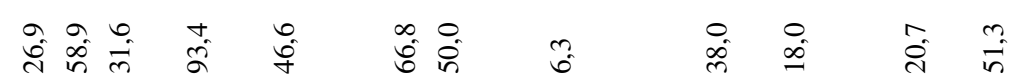

$\frac{\sqrt{2}}{3}$

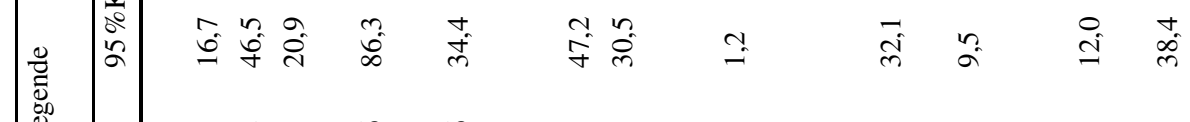

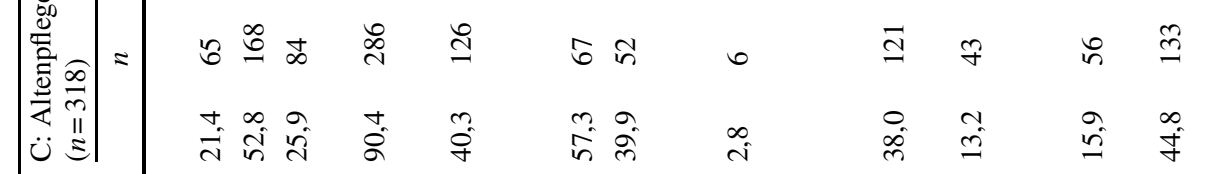

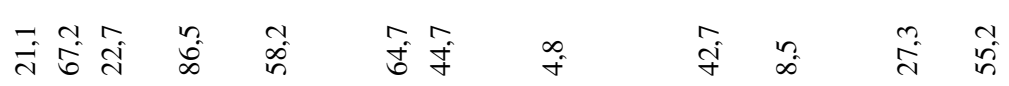

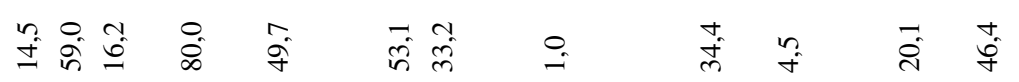

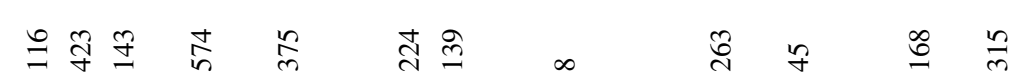

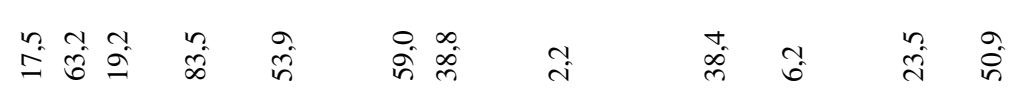

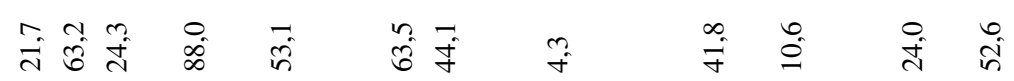

$\frac{\sqrt{2}}{8}$

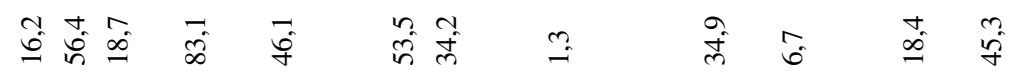

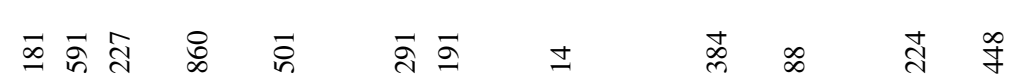




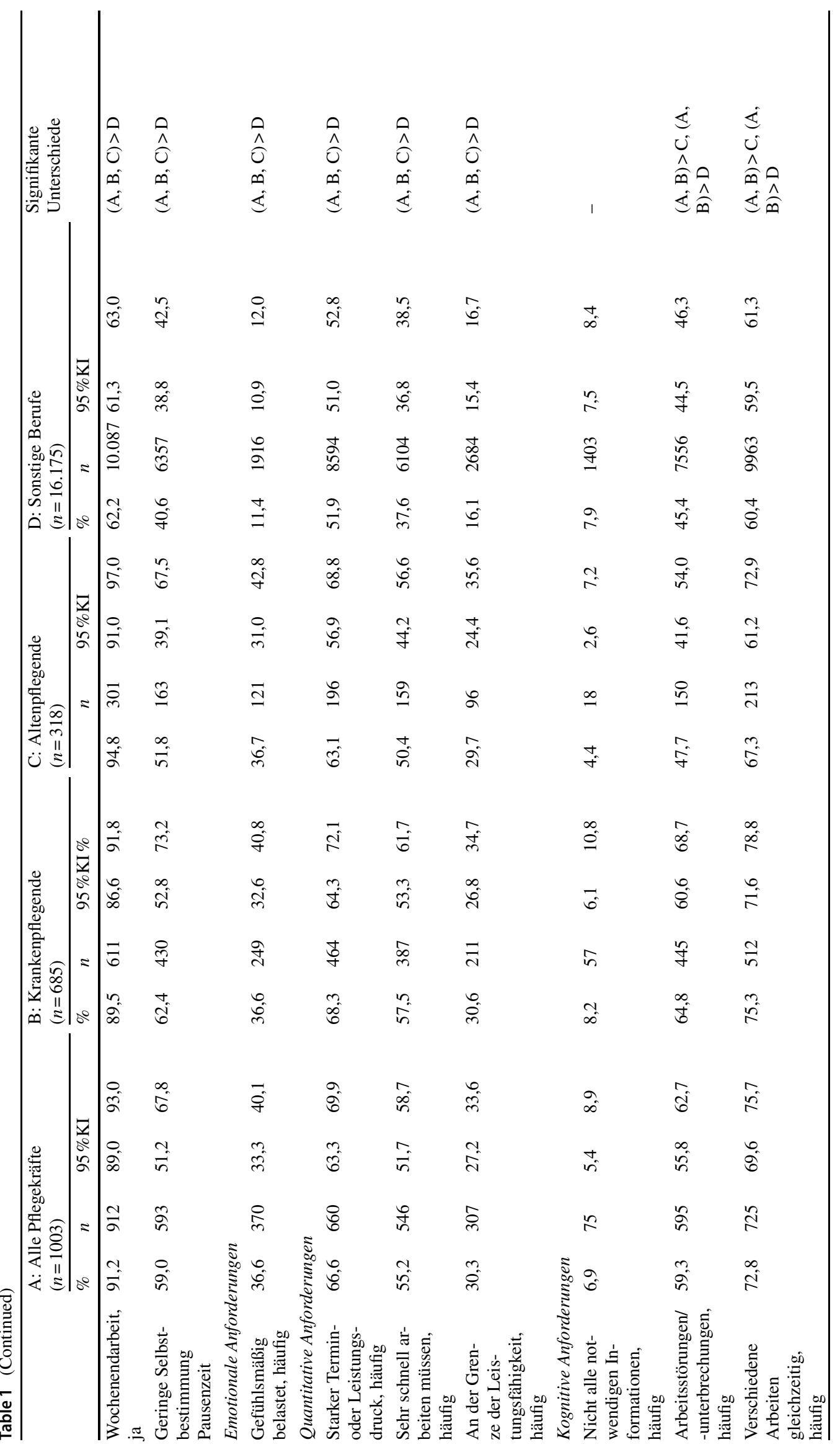




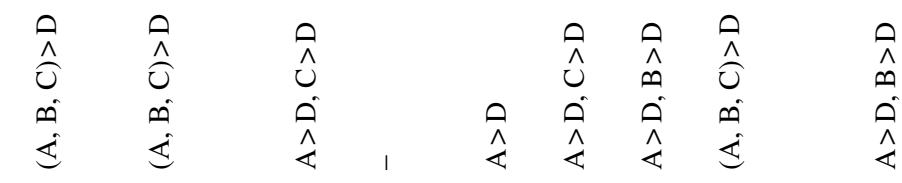

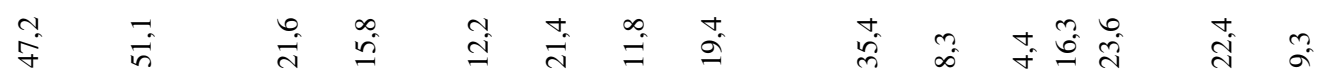

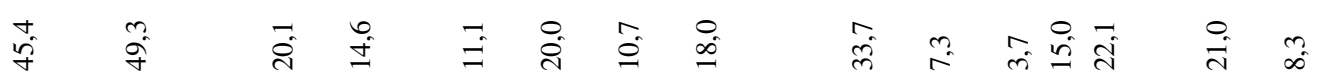

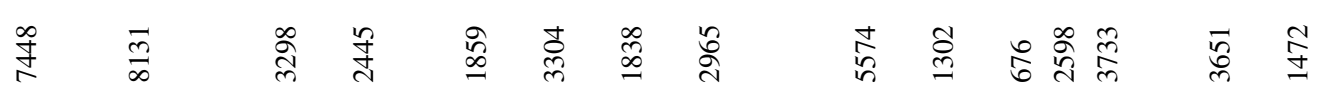

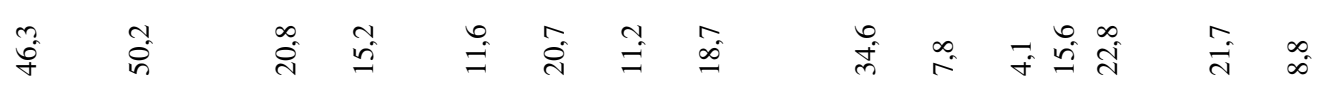

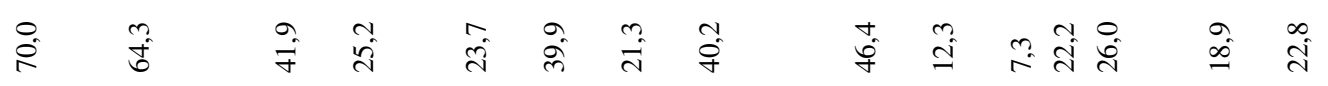

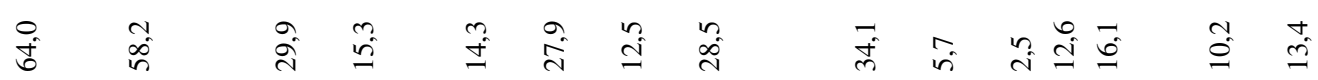

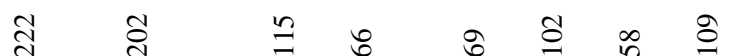

$\cong$ ำ

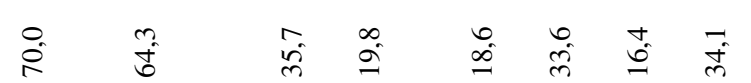

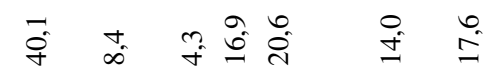

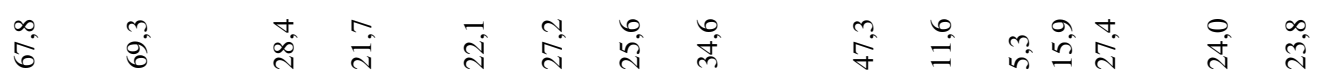

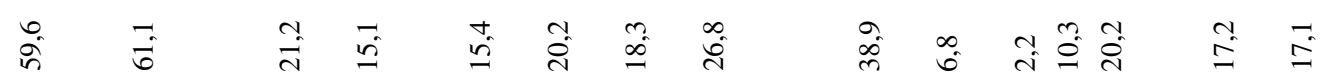
者名

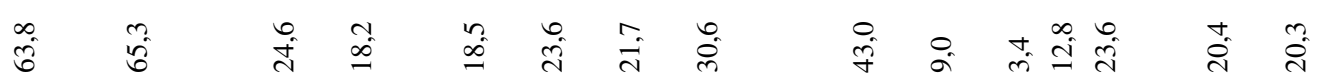

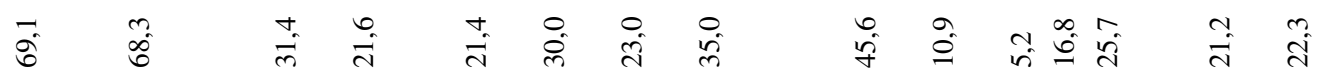

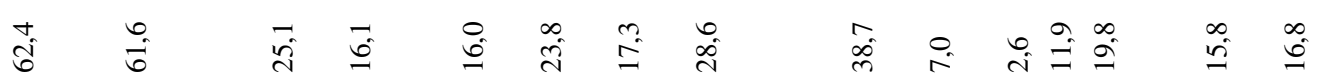

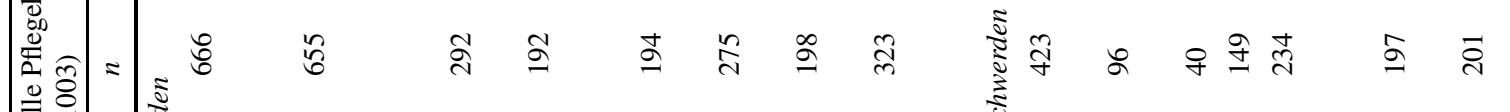

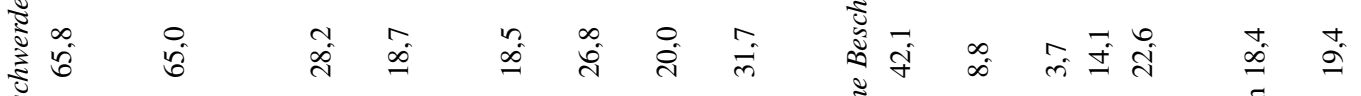

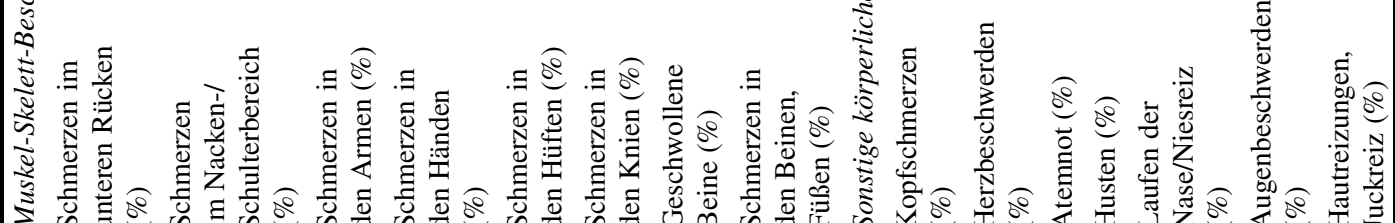




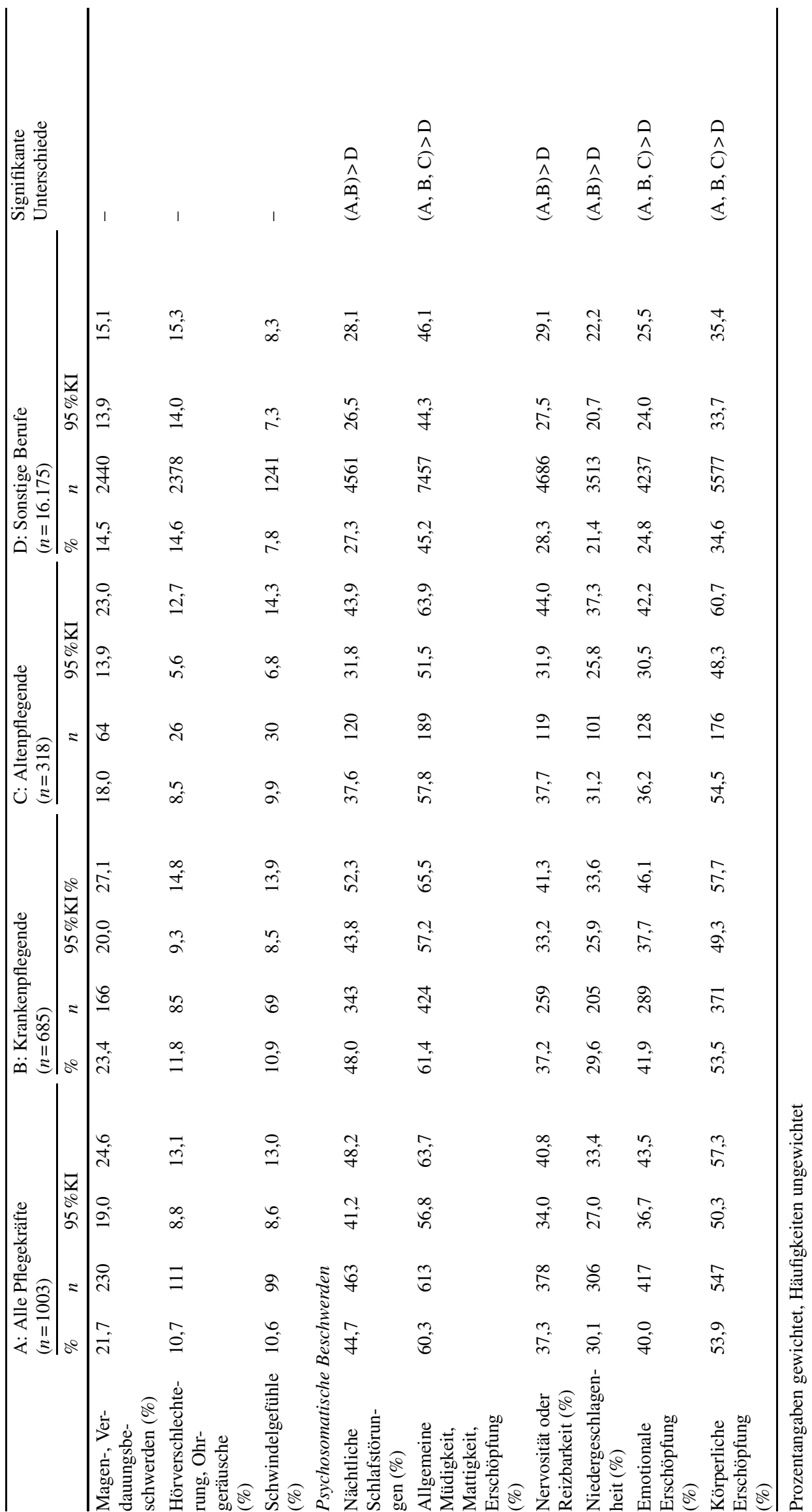




\subsection{Erhobene Variablen}

Einen detaillierten Überblick zu den Methoden und Inhalten dieser Befragung geben Rohrbach-Schmidt und Hall (2013) sowie Wittig et al. (2013). Wir beschränken uns hier auf die Vorstellung der untersuchungsrelevanten Fragen.

\subsubsection{Pausenausfall}

Der Ausfall gesetzlicher Ruhepausen wurde erfasst über die Frage „Kommt es häufig vor, dass an Arbeitstagen mit mehr als sechs Stunden Arbeitspausen ausfallen? Gemeint sind Pausen von mindestens 15 Minuten." (Antwortmöglichkeit: $0=$ nein; $1=$ ja).

\subsubsection{Unabhängige Variablen}

Gründe für den Ausfall von Ruhepausen Bei Personen, die den Ausfall von Ruhepausen bejahten, wurde mit der Frage „Woran liegt das?" und den Antwortmöglichkeiten 1 = weil ich zu viel Arbeit habe, 2 = weil Pausen nicht in den Arbeitsablauf passen oder $3=$ weil ich selbst keine Pause machen möchte, nach den individuellen Gründen gefragt.

Beschäftigungsmerkmale Mittels der Frage „Haben Sie Mitarbeiter oder Mitarbeiterinnen, für die Sie der/die direkte Vorgesetzte sind" wurde etwaig vorhandene Führungsverantwortung erhoben $(0=$ nein; $1=\mathrm{ja})$. Darüber hinaus wurde das Arbeitsvertragsverhältnis ermittelt mit dem Item ,Sind Sie gegenwärtig in einem befristeten oder in einem unbefristeten Arbeitsverhältnis?" $(1=$ befristet; $2=$ unbefristet).

Arbeitszeitmerkmale Erhoben wurde dazu die tatsächliche Arbeitszeit („Und wie lange arbeiten Sie tatsächlich im Durchschnitt in einer Woche?" Angabe in Stunden pro Woche in der Haupttätigkeit), die Schichtarbeit (umkodiertes Item „Arbeiten Sie in Schichtarbeit?“ $0=$ nein; $1=$ ja), Wochenendarbeit (zusammengefasstes Item aus „Arbeiten Sie - wenn auch nur gelegentlich - an Samstagen" mit „Arbeiten Sie - wenn auch nur gelegentlich - an Sonn-/ Feiertagen", $0=$ nein; 1=ja) und die Selbstbestimmung bei der Pausenzeit (,Wie häufig kommt es vor, dass Sie entscheiden können, wann Sie Pause machen“, 1=nie; $2=$ selten; $3=$ manchmal; $4=$ häufig).

Psychische Arbeitsanforderungen Hier wurde sowohl nach emotionalen als auch nach quantitativen und kognitiven Anforderungen der Arbeit gefragt.

Emotionale Anforderungen wurden ermittelt über die Frage „Wie häufig kommt es vor, dass Ihre Tätigkeit Sie in Situationen bringt, die Sie gefühlsmäßig belasten?" (1 = nie; $2=$ selten; $3=$ manchmal; $4=$ häufig).
Die quantitativen Anforderungen wurden erhoben über die Fragen „Wie häufig kommt es vor, dass Sie unter starkem Termin- oder Leistungsdruck arbeiten müssen?“, „Wie häufig kommt es bei Ihrer Arbeit vor, dass Sie sehr schnell arbeiten müssen?“ und „Wie häufig kommt es bei Ihrer Arbeit vor, dass Sie bis an die Grenzen Ihrer Leistungsfähigkeit gehen müssen?“ (jeweils $1=$ nie; $2=$ selten; $3=$ manchmal; $4=$ häufig).

Bei den kognitiven Anforderungen interessierten die Aspekte „Wie häufig kommt es bei Ihrer Arbeit vor, dass Sie nicht alle notwendigen Informationen erhalten, um Ihre Tätigkeit ordentlich ausführen zu können?“ und „Wie häufig kommt es bei Ihrer Arbeit vor, dass Sie bei der Arbeit gestört oder unterbrochen werden, z.B. durch Kollegen, schlechtes Material, Maschinenstörungen oder Telefonate?" sowie „Wie häufig kommt es bei Ihrer Arbeit vor, dass Sie verschiedenartige Arbeiten oder Vorgänge gleichzeitig im Auge behalten müssen?" (jeweils 1=nie; $2=$ selten; 3 = manchmal; 4 = häufig).

\subsubsection{Abhängige Variablen}

Erhoben wurden 24 gesundheitliche Beschwerden mittels der Frage ,Sagen Sie mir bitte, ob die folgenden gesundheitlichen Beschwerden bei Ihnen in den letzten 12 Monaten während der Arbeit bzw. an Arbeitstagen aufgetreten sind. Uns interessieren die Beschwerden, die häufig vorkamen" (Angabe, ob vorgekommen). Zum Teil werden die erhobenen Beschwerden im Beitrag allerdings zusammengefasst dargestellt: (1) Muskel-Skelett-Beschwerden: acht Items (Schmerzen im unteren Rücken, im Nacken-/ Schulterbereich, in den Armen, in den Händen, in den Hüften, in den Knien, in den Beinen/Füßen, geschwollene Beine), (2) sonstige körperliche Beschwerden: zehn Items (Kopfschmerzen, Herzbeschwerden, Atemnot, Husten, Laufen der Nase/Niesreiz, Augenbeschwerden, Hautreizungen/Juckreiz, Magen-, Verdauungsbeschwerden, Hörverschlechterung/Ohrgeräusche, Schwindelgefühle) und (3) psychosomatische Beschwerden: sechs Items (nächtliche Schlafstörungen, allgemeine Müdigkeit/Mattigkeit/ Erschöpfung, Nervosität/Reizbarkeit, Niedergeschlagenheit, emotionale Erschöpfung, körperliche Erschöpfung).

Die jeweils aufsummierten neu gebildeten Variablen wurden sodann anhand ihres jeweiligen Mediansplits dichotomisiert (Muskel-Skelett-Beschwerden und psychosomatische Beschwerden $=0$ wenn keine oder eine Beschwerde vorliegt, wenn mehr als eine Beschwerde vorliegt $=1$, bei sonstigen körperliche Beschwerden $=0$ wenn keine Beschwerde vorliegt, wenn mindestens eine Beschwerde vorliegt $=1$ ). 
Z. Arb. Wiss. (2019) 73:418-438

429

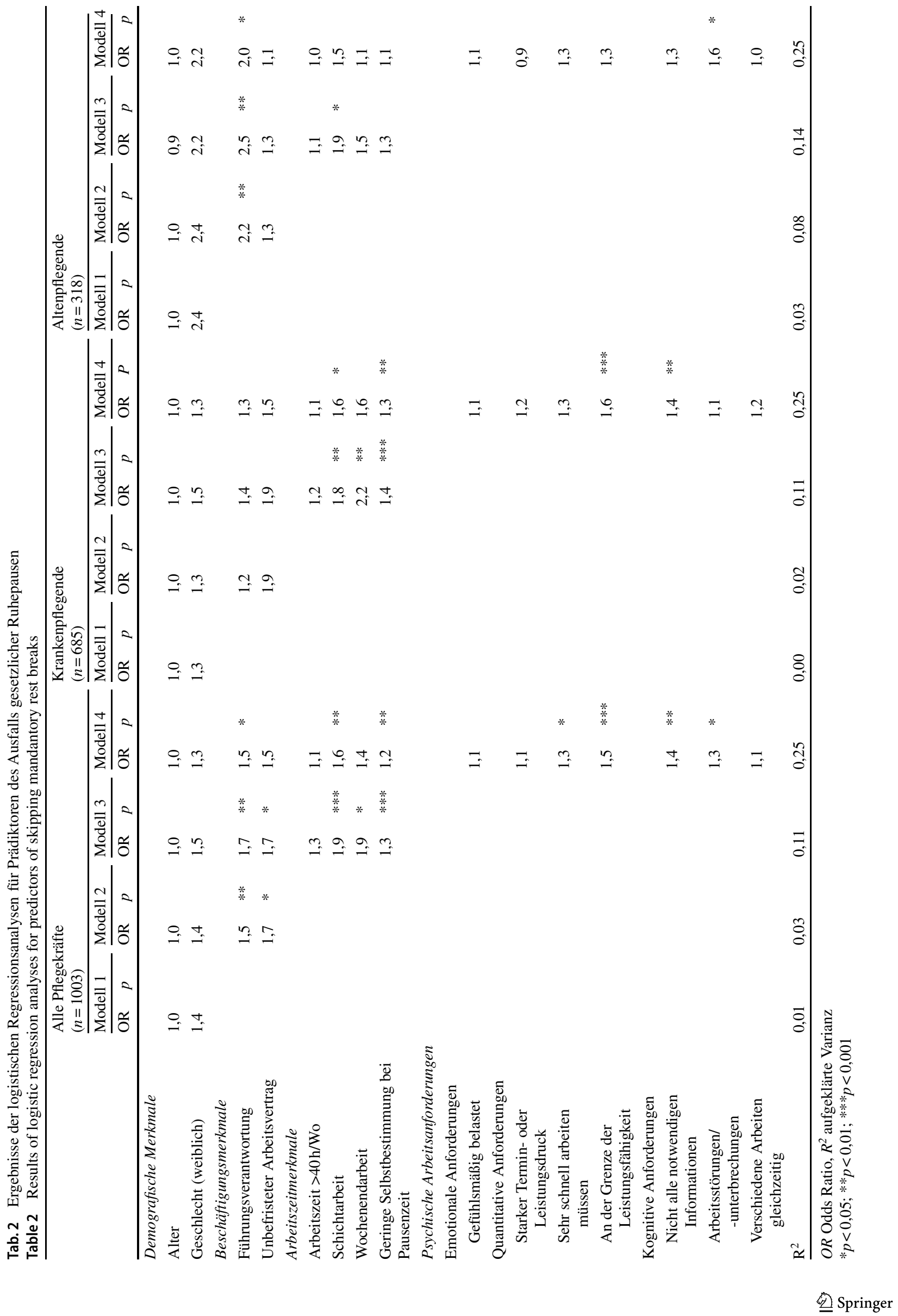




\subsection{Datenauswertung}

Die statistischen Auswertungen wurden mit der Version 24 von SPSS erstellt. Bei den Häufigkeitsanalysen zur Beschreibung der Stichproben und zur Beantwortung der Fragestellungen 1 und 2 (Vergleich Kranken- und Altenpflegende mit anderen Berufsgruppen) wurden Konfidenzintervalle für die gewichteten Prozentsätze auf Grundlage von ungewichteten Häufigkeiten berechnet. Bei überschneidenden Konfidenzintervallen unterscheiden sich die Prozentsätze bei den jeweiligen Variablen nicht bedeutsam $(p>0,05)$ zwischen den Gruppen.

Für die Untersuchung der Hypothesen 1 bis 4 und zur Beantwortung der Fragestellungen 3 und 4 (Einflussfaktoren auf Pausenausfall bei Pflegekräften) wurden binär logistische hierarchische Regressionsanalysen durchgeführt. Dabei wurden in den drei Regressionsanalysen (eine für jede Gruppe: 1. alle Pflegekräfte, 2. Krankenpflegende und 3. Altenpflegende) die unabhängigen Variablen in 4 Blöcken eingegeben (vgl. Tab. 2). Berichtet werden die Odds Ratios (OR), deren statistische Bedeutsamkeit $(p)$ und die durch das Modell aufgeklärte Varianz $\left(R^{2}\right)$.

Für die Untersuchung der Hypothese 5 (Vergleich gesundheitliche Beschwerden bei Pflegenden mit und ohne Pausenausfall) wurden ebenfalls mittels komplexer Stichproben die Konfidenzintervalle für die gewichteten Prozentsätze auf Grundlage von ungewichteten Häufigkeiten berechnet. Bei nicht überschneidenden Konfidenzintervallen wird von bedeutsamen Unterschieden $(p<0,05)$ zwischen den Gruppen ausgegangen.

Für die Analyse der Hypothese 6 wurden wieder binär logistische Regressionsanalysen durchgeführt. Dabei wurden für jede zu prognostizierende Beschwerdeform insgesamt fünf Modelle berechnet, in denen das Vorhersagemodell sukzessiv für nachfolgende Variablen erweitert wurde: Pausenausfall (= Modell 1 ), Modell 1 sowie Alter und Geschlecht (= Modell 2), Modell 2 sowie die Beschäftigungsmerkmale Führungsverantwortung und unbefristeter Arbeitsertrag (= Modell 3), Modell 3 sowie die Arbeitszeitmerkmale Arbeitszeit $>40 \mathrm{~h} / \mathrm{Wo}$, Schichtarbeit, Wochenendarbeit, geringe Selbstbestimmung bei Pausenzeit (= Modell 4), Modell 4 sowie psychische Anforderungen (Mittelwert aus emotionalen Anforderungen [gefühlsmäßig belastet], quantitativen Anforderungen [starker Termin- und Leistungsdruck, sehr schnell arbeiten müssen, an der Grenze der Leistungsfähigkeit], kognitiven Anforderungen [nicht alle notwendigen Informationen, Arbeitsstörungen/-unterbrechungen, verschiedene Arbeiten gleichzeitig]; $=$ Modell 5). Berichtet werden hier die Odds Ratios (OR) von gesundheitlichen Beschwerden durch den Ausfall von Pausen, deren Bedeutsamkeit $(p)$ und die durch das Modell aufgeklärte Varianz $\left(\mathrm{R}^{2}\right)$.

\section{Ergebnisse}

\subsection{Deskriptive Statistiken}

Hinsichtlich des Alters sind die vier Beschäftigtengruppen vergleichbar (Tab. 1). Allerdings ist in den Pflegeberufen (alle) mit 85,7\% der Anteil von Frauen erheblich höher (Krankenpflege 83,5\%, Altenpflege 90,4\%) als in anderen Berufen (51,9\%). Ebenso ist der Anteil an Führungskräften in Pflegeberufen (alle) mit 38,3\% etwas höher als in sonstigen Berufen (32,2\%), der Anteil von Beschäftigten mit einem unbefristeten Arbeitsvertrag ist hingegen ungefähr gleich.

Der Prozentsatz von Beschäftigten mit regelmäßigen, tatsächlichen wöchentlichen Arbeitszeiten über 40 Stunden ist in den Pflegeberufen geringer $(21,1 \%$, kein bedeutsamer Unterschied zwischen Kranken- und Altenpflege) als in anderen Berufen $(34,7 \%)$, was vermutlich in einer höheren Teilzeitquote begründet liegt. Hingegen ist der Prozentsatz an Wochenendarbeit (Pflegeberufe 91,2\%, sonstige 62,2\%) und Schichtarbeit (Pflegeberufe 48,9\%, sonstige 10,2\%) im Vergleich weitaus höher (kein bedeutsamer Unterschied zwischen Kranken- und Altenpflege). Auch eine geringe Selbstbestimmung bezüglich des Pausenzeitpunktes wird in Pflegeberufen häufiger berichtet $(59,0 \%$, kein bedeutsamer Unterschied zwischen Kranken- und Altenpflege) als in anderen Berufen (40,6\%).

Die psychischen Anforderungen sind in Pflegeberufen fast ausnahmslos höher als in sonstigen Berufen (auBer mangelnde Informationen). Bei den Arbeitsunterbrechungen und dem Multitasking (verschiedene Arbeiten gleichzeitig) liegen die psychischen Anforderungen in der Krankenpflege allerdings noch über denen der Altenpflege (Tab. 1).

\subsection{Häufigkeit und Gründe des Pausenausfalls}

Hinsichtlich des Pausenausfalls und der Fragestellung 1 ist festzustellen (s. Tab. 1), dass in Pflegeberufen von weitaus mehr Beschäftigten häufiger Pausenausfall angegeben wird $(49,6 \%)$ als von Beschäftigten anderer Berufe $(26,0 \%)$. Dies gilt dabei in bedeutsam höherem Maße für die Krankenpflege $(53,9 \%)$ als für die Altenpflege $(40,3 \%)$. Während jedoch in Berufen außerhalb der Pflege vor allem die mangelnde Passung in den Arbeitsablauf als überwiegender Grund für den Pausenausfall (Fragestellung 2) angegeben wird $(48,3 \%)$, ist es bei den Pflegeberufen vor allem zu viel Arbeit $(58,6 \%)$. Dies trifft sowohl für die Krankenpflege $(59,0 \%)$ als auch für die Altenpflege $(57,3 \%)$ zu (sonstige Berufe 37,0\%). Am wenigsten liegt es daran, dass Beschäftigte selbst keine Pausen machen möchten, dies gilt für alle Gruppen (Pflegeberufe: $2,4 \%$, andere: 14,7\%; s. Tab. 1). 


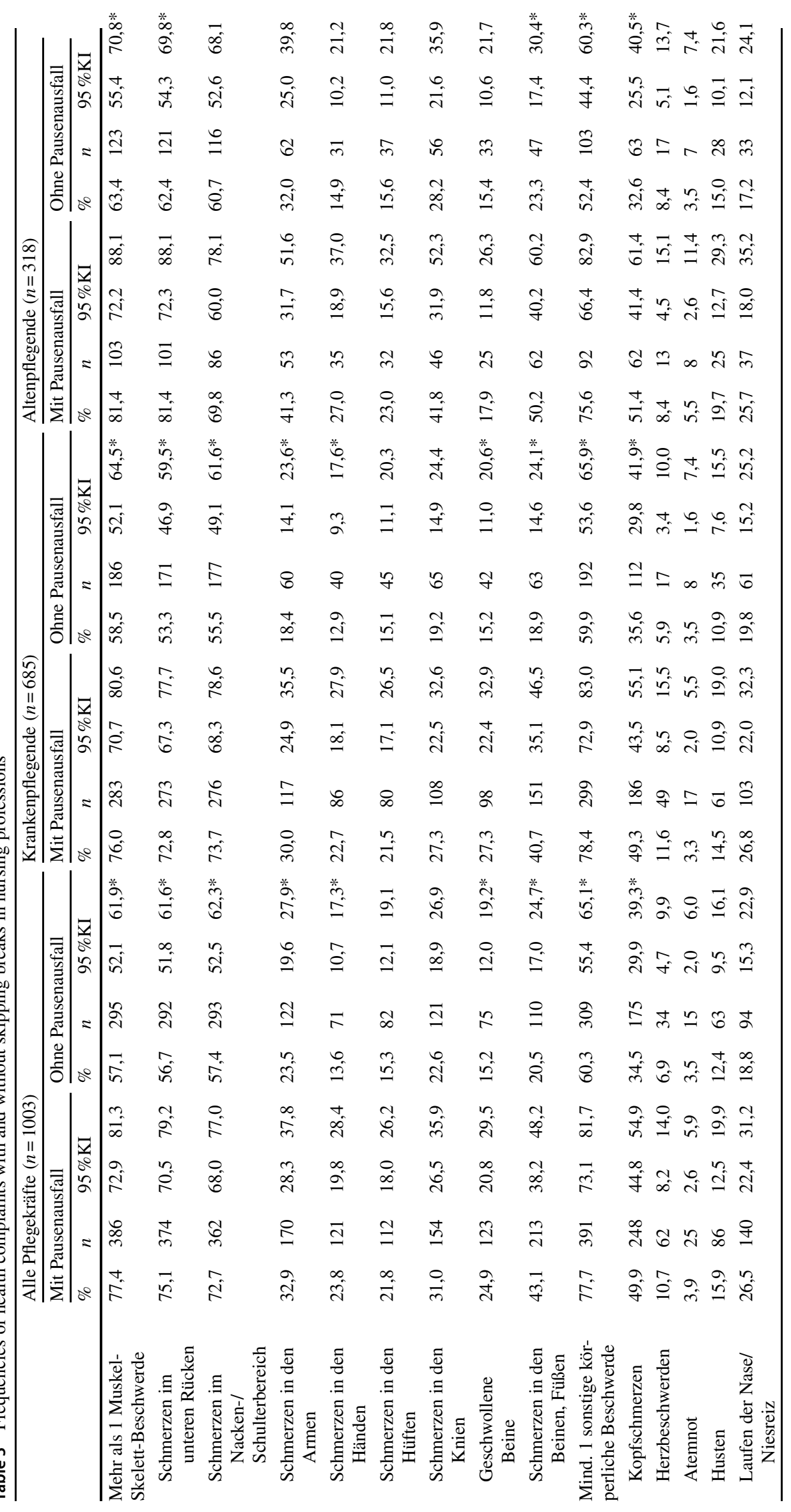




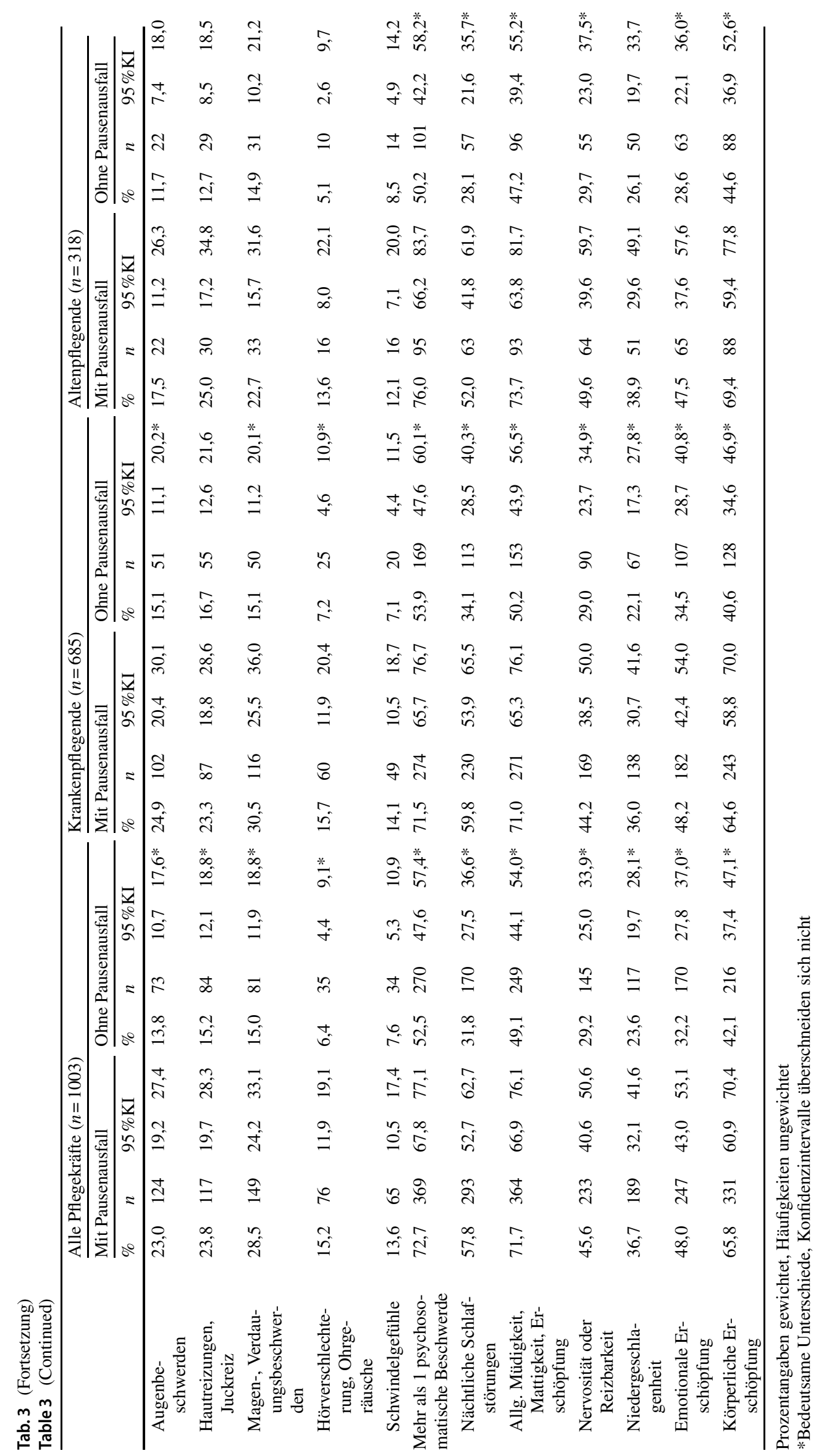




\subsection{Prädiktoren des Ausfalls gesetzlicher Ruhepausen}

Für die demografischen Variablen Alter und Geschlecht ist in der logistischen Regressionsanalyse kein bedeutsamer Effekt auf den Pausenausfall (ohne Hypothese) festzustellen (Tab. 2). Allerdings zeigt sich, dass bei Führungskräften in der Pflege insgesamt $(\mathrm{OR}=1,5)$ sowie in der Altenpflege $(\mathrm{OR}=2,0)$ das Risiko für den Pausenausfall signifikant höher ausfällt als bei Beschäftigten ohne Führungsverantwortung (Hypothese 1). In der Krankenpflege ist dieser Zusammenhang $(\mathrm{OR}=1,3)$ allerdings statistisch nicht abzusichern. Der unterschiedliche Vorhersagebeitrag der Führungsverantwortung für den Pausenausfall in beiden Berufsgruppen ist signifikant $(\mathrm{z}=1,73, p=0,048)$. Das Beschäftigungsverhältnis - Befristung versus Festanstellung - hat hingegen im letzten Schritt der Regression in keiner der drei untersuchten Gruppen bedeutsamen Einfluss auf die Häufigkeit des Pausenausfalls (Fragestellung 3).

Hinsichtlich der psychischen Arbeitsanforderungen zeigt sich in der Gesamtstichprobe der Pflegekräfte ein höheres Risiko für den Pausenausfall für die Merkmale (Hypothese 2) "sehr schnell arbeiten müssen“ $(\mathrm{OR}=1,3)$,, an der Grenze der Leistungsfähigkeit arbeiten“ ( $\mathrm{OR}=1,5)$, „nicht alle notwendigen Informationen haben" $(\mathrm{OR}=1,4)$ und „Arbeitsstörungen/-unterbrechungen“ $(\mathrm{OR}=1,3)$. Dabei ist in der Krankenpflege ein höheres Risiko für den Aspekt ,an der Grenze der Leistungsfähigkeit“ (Odds Ratio $=1,6)$ und „nicht alle notwendigen Informationen“ $(\mathrm{OR}=1,4) \mathrm{zu}$ finden, in der Altenpflege für „Arbeitsstörungen/-unterbrechungen" $(\mathrm{OR}=1,6)$.

Für die wöchentliche Arbeitszeit über 40h (Hypothese 3) tritt in keiner Gruppe ein signifikanter Zusammenhang mit dem Pausenausfall auf, ebenso wenig wie für die Wochenendarbeit (Fragestellung 4b). Schichtarbeit als ein weiteres Arbeitszeitmerkmal (Fragestellung 4a) birgt hingegen für die Pflege insgesamt und insbesondere für Krankenpflegende ein höheres Risiko für den Pausenausfall $(\mathrm{OR}=1,6)$. Ein geringer zeitlicher Entscheidungsspielraum bei der Pausennahme (Hypothese 4) geht in der Gruppe der Pflegekräfte insgesamt $(\mathrm{OR}=1,2)$ und bei Krankenpflegenden $(\mathrm{OR}=1,3)$ mit einem erhöhten Risiko für den Ausfall von Pausen einher, nicht jedoch bei Altenpflegenden.

\subsection{Wirkungen des Ausfalls gesetzlicher Ruhepausen auf berichtete Beschwerden}

Zunächst ist festzustellen, dass gesundheitliche Beschwerden signifikant häufiger bei Pflegekräften auftreten, die den Ausfall von Ruhepausen berichten als bei denen, die dies nicht tun (Tab. 3: mehr als 1 Muskel-Skelett-Beschwerde: $77,4 \%$ vs. $57,1 \%$; mindestens eine sonstige körperliche Be-

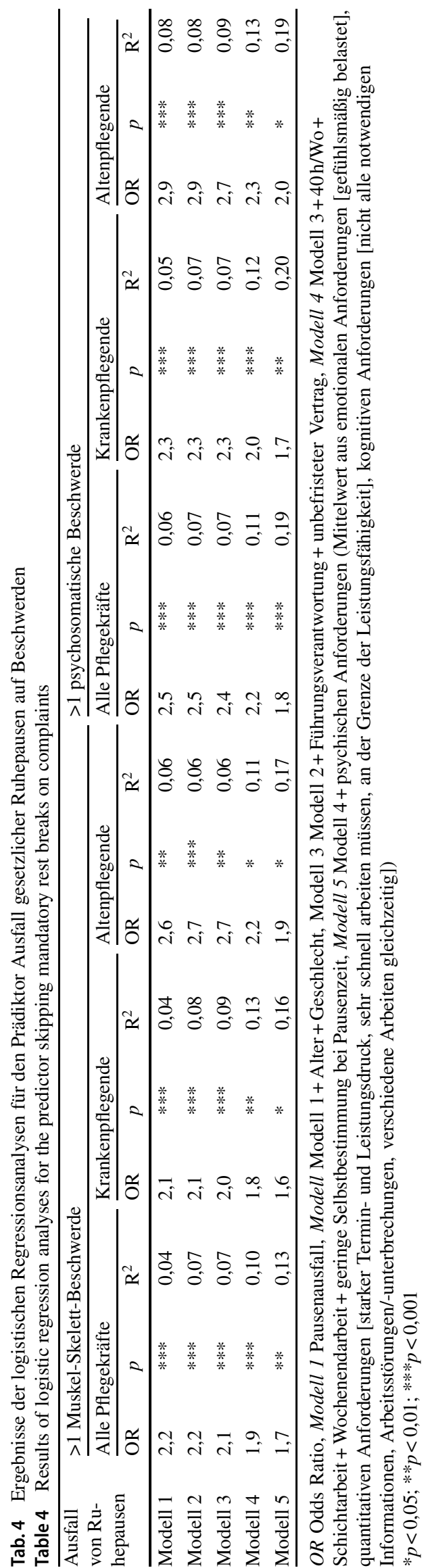


schwerde: $77,7 \%$ vs. $60,3 \%$; mehr als 1 psychosomatische Beschwerde: $72,7 \%$ vs. $52,5 \%$ ).

Für alle drei Gruppen gilt, dass vor allem psychosomatische Beschwerden öfter bei häufigem Pausenausfall angegeben werden (Hypothese 6). Bei den körperlichen Beschwerden (Hypothese 5) werden vor allem Muskel-SkelettBeschwerden häufiger bei Pausenausfall angegeben als ohne.

Selbst bei Kontrolle von demografischen Merkmalen (Alter, Geschlecht), Beschäftigungsmerkmalen (Führungsposition, unbefristeter Vertrag), Arbeitszeitmerkmalen (Arbeitszeit $>40 \mathrm{~h} /$ Wo, Schichtarbeit, Wochenendarbeit, geringe Selbstbestimmung bei Pausenzeit) und psychischen Anforderungen (Mittelwert aus emotionalen Anforderungen [gefühlsmäßig belastet], quantitativen Anforderungen [starker Termin- und Leistungsdruck, sehr schnell arbeiten müssen, an der Grenze der Leistungsfähigkeit], kognitiven Anforderungen [nicht alle notwendigen Informationen, Arbeitsstörungen/-unterbrechungen, verschiedene Arbeiten gleichzeitig]) erhöht der berichtete Pausenausfall noch signifikant das Risiko sowohl für Muskel-Skelett- als auch für psychosomatische Beschwerden (Tab. 4).

\section{Diskussion}

Unsere Ergebnisse zeigen, dass Pausen in Pflegeberufen häufiger ausfallen als in anderen Berufen (Fragestellung 1). $\mathrm{Zu}$ hohe quantitative Anforderungen (,zu viel zu tun“) ist dabei im Pflegebereich der häufigste Grund für Pausenausfall, er wird hier auch häufiger angegeben als in anderen Berufen (Fragestellung 2). Hypothese 1 kann nur zum Teil bestätigt werden, denn nur in der Altenpflege lassen Führungskräfte die Pausen häufiger ausfallen als Beschäftigte ohne Mitarbeiterverantwortung, nicht jedoch in der Krankenpflege. Eine Befristung des Arbeitsverhältnisses hängt mit dem Pausenausfall nicht zusammen (Fragestellung 3). Auch Hypothese 2 kann nur teilweise bestätigt werden, denn psychische Anforderungen (schnell arbeiten müssen, Arbeiten bis zur Grenze der Leistungsfähigkeit, fehlende Informationen und Arbeitsunterbrechungen) gehen mit einem häufiger berichteten Pausenausfall einher, gefühlsmäßige Belastung, Termin- und Leistungsdruck und Multitasking dagegen nicht. Hypothese 3 ist hingegen abzulehnen: Lange Arbeitszeiten tragen in Pflegeberufen nicht zum Pausenausfall bei. Allerdings macht es einen Unterschied, ob der Pausenzeitpunkt selbst bestimmt werden kann oder nicht (Hypothese 4): Bei geringer Selbstbestimmung steigt das Risiko für den Pausenausfall. Dies ist ebenso für Schichtarbeitende der Fall (Fragestellung 4a), nicht hingegen für Wochenendarbeitende (Fragestellung 4b). Das Risiko für körperliche (Hypothese 5) und vor allem für psychosomatische (Hypothese 6) Beschwerden ist höher, wenn Pausen ausfallen, selbst bei Kontrolle anderer Beschäftigungs- und Arbeitsmerkmale.

\subsection{Theoretische Implikationen}

In der Erholungs- und speziell der Pausenforschung wurden bisher überwiegend Wirkungen von Arbeitspausen untersucht (Wendsche und Lohmann-Haislah 2018). Als moderierende Faktoren standen dabei insbesondere Merkmale der Pausenorganisation, wie die Länge, die Verteilung und die Lage der Pausen sowie die von den Beschäftigten genutzten Pausentätigkeiten im Fokus. Wenig betrachtet wurde bisher die Rolle länderspezifischer Regelungen für die Pausenorganisation und die tatsächliche Umsetzung dieser Vorgaben. Unsere Studie erweitert daher die Forschungsperspektive, indem auch Einflussfaktoren und Wirkungen der Nichteinhaltung von Mindestvorgaben an längere Ruhepausen im Kontext der Arbeit von Pflegekräften in Deutschland genauer untersucht wurden.

Es ist zunächst festzuhalten, dass solche Pausenregeln in unserer Stichprobe nur bedingt eingehalten werden. Unabhängig vom Beruf berichtet mehr als jeder vierte Beschäftigte in Deutschland, dass gesetzliche Ruhepausen regelmäßig ausfallen. Allerdings zeigt unsere Studie auch, dass die Risiken für den Pausenausfall in Pflegeberufen substanziell erhöht sind. Für die Erholungs- und Pausenforschung bedeutet dies, dass zukünftig auch stärker Kontextfaktoren (z.B. Berufsgruppen) sowie weiter gefasste Operationalisierungen von Erholungsverhalten in die Theoriebildung integriert werden müssen (Sonnentag et al. 2017).

Nur ein geringer Anteil an Pflegekräften berichtet in unserer Studie individuelle Gründe für den Ausfall von Ruhepausen. Demografische Merkmale wie Alter und Geschlecht erklärten keine substanzielle Varianz im Pausenverhalten. Dies deckt sich mit Befunden aus anderen Pausenstudien in der Pflegeforschung (Wendsche et al. 2017) sowie der Forschung zum Erholungserleben von Beschäftigten (Wendsche und Lohmann-Haislah 2017a, 2017b). Im Einklang mit theoretischen Modellen der Erholungsforschung (Meijman und Mulder 1998; Sonnentag et al. 2017) sind es daher eher arbeits-, organisations- und rollenspezifische Merkmale, die das Erholungsverhalten der Beschäftigten bestimmen.

Im Einklang mit rollen- und austauschtheoretischen Annahmen zum Mitarbeiterverhalten (Blau 1964; Gouldner 1960; Katz und Kahn 1978) gab es durchaus Hinweise, dass Führungsverantwortung, insbesondere bei Altenpflegekräften, unabhängig von demografischen Merkmalen, Arbeitszeitmerkmalen und psychischen Arbeitsanforderungen mit erhöhten Risiken für den Ausfall von Ruhepausen einhergeht. Zukünftige Untersuchungen müssten dabei jedoch stärker die beteiligten Mechanismen ins Blickfeld nehmen, da höhere Arbeitslasten als alleinige Erklärung nicht aus- 
reichen. Von Interesse ist dabei, ob bei Führungskräften eine durch Personalauswahlprozesse oder individuelle Rollenzuschreibungen überformte höhere individuelle Verausgabungsneigung, höhere Leistungsbereitschaft sowie reduzierte Erholungsfähigkeit das Ausfallenlassen von Pausen erklären. Genauer zu untersuchen ist auch, wie sich das Pausenverhalten der Führungskräfte auf Beschäftigte auswirkt, z. B. durch gruppenspezifische Erholungs- und Pausennormen und ein ungünstiges Erholungsklima im Betrieb. Wir fanden zusätzlich Hinweise, dass Pflegekräfte mit unbefristeten Arbeitsverhältnissen im Vergleich zu befristet Beschäftigten erhöhten Risiken ausgesetzt sind, Ruhepausen ausfallen zu lassen. Diese Zusammenhänge lassen sich allerdings nicht in den einzelnen Pflegeberufen statistisch absichern, womöglich auch aufgrund geringer Basisraten befristeter Pflegekräfte. Zudem verschwinden sie, wenn psychische Arbeitsanforderungen im Vorhersagemodell berücksichtigt werden. Dies deutet darauf hin, dass eine höhere psychische Arbeitsbelastung statt gruppenpsychologische Prozesse diese Beziehungen vermittelt. Dies bedarf weiterer Abklärung, auch in anderen Berufsgruppen, da in anderen Ländern konträre Belastungssituationen in Abhängigkeit vom Arbeitsvertrag gefunden wurden (Saloniemi et al. 2004).

Als zentrale Risikofaktoren für den Ausfall von Ruhepausen bei Pflegekräften identifizierten wir hier hohe quantitative (schnelles Arbeiten, Arbeiten an der Leistungsgrenze) und kognitive (Informationsmängel, Arbeitsunterbrechungen) Arbeitsanforderungen. Dies deckt sich mit den Ursachenzuschreibungen der Pflegekräfte und den Annahmen des Rahmenmodells der Arbeitsintensität (Rau und Göllner 2018). Dieses postuliert, dass das Ausfallenlassen von Pausen ein zeitsparendes Bewältigungsverhalten von Arbeitssituationen darstellt, welches durch eine begrenzte Zeit zur Auftragserfüllung bei hohen quantitativen und kognitiven Regulationsanforderungen gekennzeichnet ist. Alternative theoretische Ansätze (Challenge-Hindrance-Stressor-Modell; Cavanaugh et al. 2000), die stark motivierende Arbeitsmerkmale als Ursachen für ein verändertes Zeiterleben während der Arbeit (z.B. subjektive Unterschätzung der Belastungsdauer) sowie die Einschätzung einer verminderten Notwendigkeit von Pausen annehmen, scheinen damit als alleinige Erklärung für den Ausfall von Ruhepausen bei Pflegekräften auszuscheiden. Allerdings gibt es auch hier Hinweise auf Bedeutungsunterschiede dieser Risikofaktoren in Abhängigkeit vom Pflegeberuf (Kranken- vs. Altenpflege). Unterschiede in der Prävalenz riskanter Ausprägungen dieser Belastungsfaktoren scheiden nach unseren Daten als Ursache eher aus. Zukünftige Untersuchungen sollten deshalb genauer untersuchen, welche Rolle spezielle kognitive und quantitative Arbeitsanforderungen im Zusammenspiel mit dem Pausenverhalten für die Auftragsbewältigung von Kranken- und Altenpflegekräften spielen.
Die Befundlage zu Zusammenhängen zwischen Arbeitszeit- und Pausengestaltung bei Pflegekräften war bisher inkonsistent (Wendsche et al. 2017). Unsere Ergebnisse deuten darauf hin, dass für die Vorhersage des Ausfalls von Ruhepausen weniger die Gesamtbelastungsdauer (Länge der Arbeitszeit) sondern vielmehr die Lage der Arbeitszeit entscheidend ist. Schichtarbeit ist bei Pflegekräften unabhängig von demografischen Merkmalen, Beschäftigungsmerkmalen und psychischen Arbeitsanforderungen mit erhöhten Risiken des Ausfalls von Ruhepausen assoziiert. Dies bedeutet, dass negative gesundheitliche Wirkungen von Schichtarbeit nicht nur über eingeschränkte Erholungsmöglichkeiten während der Ruhezeit (z. B. Schlafprobleme; Amlinger-Chatterjee 2016), sondern eventuell auch zusätzlich über ein ungünstigeres Erholungsverhalten während der Arbeit erklärt werden können. Die Annahme einer kumuliert eingeschränkten Erholung bei Schichtarbeitern sollte zukünftig weiter geprüft werden. Zu klären sind auch die beteiligten Mechanismen für erhöhte Risiken von Pausenausfällen bei im Schichtsystem arbeitenden Pflegekräften. Im Pflegekontext sind dabei Unterschiede in der Personalbemessung und der damit einhergehenden Möglichkeit von Springer- und Vertreterregelungen während Pausen (möglicherweise Erschwernisse in Nachtschichten) von Interesse. Unabhängig von der Schichtarbeit als Risikofaktor für den Pausenausfall fanden wir auch Hinweise für höhere Prävalenzraten des Ausfalls von Ruhepausen bei Pflegekräften, die am Wochenende arbeiten müssen. Unsere Daten legen allerdings nahe, dass dies durch erhöhte psychische Arbeitsbelastung erklärt werden kann.

Eine neue Erkenntnis aus unseren Ergebnissen betrifft die Senkung des Risikos von Pausenausfällen, wenn Pflegekräfte ausreichend zeitliche Freiheitsgrade über die Pausenorganisation haben. Dies mag in erster Sicht kontraintuitiv wirken. Allerdings ist zu berücksichtigen, dass gewisse zeitliche Spielräume hinsichtlich der Auslösung längerer Pausen durchaus das Beendigen von Arbeitsaufträgen (i.S. Versorgung von Klienten) fördern dürften und damit einer Unterbrechung der Ruhepause vorbeugen. Zusätzlich zeigte eine Beobachtungsstudie, dass in Pflegeteams mit regelmäßigeren Pausen auch eher kollegiale Gruppenpausen möglich sind (Wendsche et al. 2014). Die Vorbeugung von Pausenunterbrechungen durch zeitliche Freiheitsgrade bei der Pausenorganisation unterstützt damit auch den sozialen Austausch zwischen den Beschäftigten und damit die Erfüllung eines zentralen Pausenmotivs von Beschäftigten (Wendsche und Lohmann-Haislah 2018).

Im Einklang mit theoretischen Annahmen der Belastungs-, Beanspruchungs- und Erholungsforschung (Graf et al. 1970; Meijman und Mulder 1998; Sonnentag et al. 2017) sowie empirischen Befunden aus der Pflegeforschung (Wendsche et al. 2017), finden wir in unseren Daten auch, dass die Einhaltung von Ruhepausen bei Pflegekräften 
mit einem reduzierten Risiko für körperliche und psychosomatische Beschwerden einhergeht. In Erweiterung vorheriger Analyseansätze können wir allerdings hier den Einfluss anderer Faktoren wie demografische Merkmale, den Pflegeberuf sowie Beschäftigungs-, Arbeitszeit- und Arbeitsanforderungsmerkmale auf diese Zusammenhänge ausschließen. Unsere Ergebnisse zeigen damit, dass gesetzliche Ruhepausen geeignet sind, negative körperliche und psychische Beanspruchungsfolgen abzubauen.

\subsection{Limitationen der Studie und Aufgaben für zukünftige Forschung}

Sicherlich stellen die repräsentative Auswahl der Gesamtstichprobe und der Stichprobenumfang Stärken unserer Untersuchung dar, insbesondere hinsichtlich der Verallgemeinerbarkeit der dargestellten Ergebnisse und der hohen Teststärke bei der Prüfung unserer Annahmen. Zu berücksichtigen ist allerdings die beschränkte Repräsentation von Altenpflegekräften, die möglicherweise dazu beitrug, dass kleinere und durchaus hypothesenkonforme Effekte hier keine statistische Absicherung erreichen konnten.

Auf der anderen Seite sind auch einige Limitationen offensichtlich. Dies betrifft beispielweise die Studienanlage im Querschnitt, welche Aussagen über Zusammenhänge, nicht jedoch von kausalen Wirkbeziehungen ermöglicht. Eine Option dürfte die Wiederholung der dargestellten Analysen mit den Daten der von der Bundesanstalt für Arbeitsschutz und Arbeitsmedizin prospektiv angelegten Arbeitszeitbefragung sein (Wöhrmann et al. 2016), wenn der Datensatz der 2. Erhebungswelle vorliegt. Eine andere Begrenzung liegt in der für Surveybefragungen typischen Nutzung von 1-Item-Maßen zur Abbildung von Konstrukten, auch um eine hohe Compliance bei der Telefonbefragung zu sichern. Dieses Vorgehen kann allerdings Einschränkungen in der Messgenauigkeit nach sich ziehen. In Folge sind die hier dargestellten Zusammenhänge zwischen den Variablen eher konservative Schätzer. Dies könnte auch erklären, warum beispielsweise nicht alle Facetten der quantitativen Arbeitsanforderungen in unserer Untersuchung als Prädiktoren für Pausenausfall identifiziert werden konnten. So wäre es in zukünftigen Untersuchungen sinnvoll aus den 1-Itemmaßen latente Konstrukte zu bilden.

$\mathrm{Zu}$ berücksichtigen ist auch, dass die hier abgebildeten Variablen eher zeitlich stabile Ausprägungen abbilden. In der Forschung wurde allerdings gezeigt, dass Erholungsverhalten und Erholungserleben auch zeitlich kürzeren Dynamiken unterliegt, woraus sich der Bedarf ergibt, unsere Annahmen auch mittels Tagebuchstudien erneut zu prüfen (Sonnentag et al. 2017).

Eine wesentliche Einschränkung solcher Surveystudien liegt darin, dass Besonderheiten von Berufsgruppen und Arbeitsbedingungen nur begrenzt abgebildet werden können.
Dies betrifft wichtige Einflussgrößen auf die Pausenorganisation im Pflegekontext in mehrfacher Hinsicht. So wurden in anderen Untersuchungen Unterschiede in Abhängigkeit von der Pflegeart (Wendsche et al. 2014) und vom Pflegekontext (Wendsche et al. 2017) berichtet. Dem Einfluss solcher übergeordneter Einflussgrößen auf die Pausenorganisation sollte in Zukunft stärker nachgegangen werden, u.a. auch deshalb, weil diese zukünftig eine unterschiedliche Bedeutsamkeit für die Versorgungssituation von Pflegebedürftigen haben dürften (Zunahme mobiler Pflegeformen) und damit kontextspezifische Präventions- und Interventionsstrategien für den Arbeits- und Gesundheitsschutz von Pflegekräften nötig werden.

\subsection{Praktische Implikationen}

Unsere Ergebnisse implizieren spezifische Handlungsaufforderungen an die Arbeitsaufsicht und Politik sowie an Pflegeleitungen und Pflegekräfte, um die Pausenorganisation in Pflegeberufen nachhaltig zu verbessern.

Nahezu die Hälfte der befragten Pflegekräfte berichten hier Verstöße gegen die Pausenvorgaben laut Arbeitszeitgesetz ( $\$ 4)$, vor allem aus arbeitsorganisatorischen und weniger aus individuellen Gründen. Insofern sollten Aufsichtsbehörden die Arbeitszeitkontrollen in dieser Berufsgruppe verstärken und Gesetzesverstöße konsequent ahnden.

Die Ergebnisse unserer Studie zeigen, dass die Einhaltung gesetzlicher Ruhepausen bei Pflegekräften mit weniger körperlichen und psychosomatischen Beschwerden einhergeht und zwar unabhängig von den Wirkungen andere Beschäftigtenmerkmale sowie anderer Arbeits- und Organisationsmerkmale. Diese Befunde untersetzen den besonderen Regelungsbedarf von Arbeitspausen im Arbeitszeitgesetz und deren wichtige Rolle im Gesundheitsschutz entsprechend dem Arbeitsschutzgesetz. Nicht nur Pflegekräfte dürften ganz persönlich in Form eines besseren Wohlbefindens von der Einhaltung der Ruhepausen profitieren, sondern insbesondere auch die Arbeitgeber in diesem Berufsfeld. So verweisen unsere Befunde auf möglicherweise reduzierte Risiken für krankheitsbedingte Personalausfälle bei Einhaltung von Pausen. Fehlende Pausen wurden in anderen Pflegestudien auch in Zusammenhang mit häufigeren Pflegefehlern und einem höheren Risiko für Fachkraftabgänge gebracht (Wendsche et al. 2017). Insofern ist es nötig, dass Pflegeleitungen und Pflegekräfte gemeinsam handlungswirksame Strategien zur Reduktion des Ausfalls von Ruhepausen entwickeln. Welche Ansatzpunkte ergeben sich nun dabei aus unseren Daten?

Eine besondere Rolle kommt dabei den Führungskräften zu, die hier häufiger den Ausfall von Pausen berichten. Führungskräfte sollten deshalb selbstkritisch ihr eigenes Erholungsverhalten beobachten, hinterfragen und gegebenenfalls optimieren. Dies ist insofern wichtig, als dass das Füh- 
rungskräfteverhalten ein Rollenmodell für die Beschäftigten liefern kann und sich erholungsungünstige Gruppennormen entwickeln können. Bestimmte Formen der Arbeitszeitorganisation, die Risiken für den Ausfall von Pausen in sich bergen, wie beispielsweise Schicht- und Wochenendarbeit, sind überwiegend Kern des Pflegeberufes. Gerade aber bei der Arbeit mit atypischen Arbeitszeiten muss zusätzlicher Arbeitsbelastung vorgebeugt werden. $\mathrm{Zu}$ hinterfragen ist hier vor allem, wie eine ausreichende Personalbemessung und angemessene Arbeitsdichte während solcher Arbeitsschichten abgesichert werden kann. Mit einer Unterbesetzung oft einhergehende Merkmale hoher Arbeitsintensität wie hohes Arbeitstempo, die Notwendigkeit einer hohen Verausgabung, Informationsmängel sowie Arbeitsunterbrechungen (Glaser und Höge 2005) stellten sich als zentrale Prädiktoren des Ausfalls von Ruhepausen in unserer Studie heraus. Die Optimierung solcher Arbeitsbelastungsfaktoren ist demnach eine zentrale Ansatzstelle, um eine ausreichende Erholung von Pflegekräften zu gewährleisten.

Pflegkräfte sollten zudem auch bestimmte zeitliche Freiheitsgrade bei der Pausenauslösung haben. Pausenvorgaben in Zeitfenstern sind dabei das Gestaltungsmittel der Wahl, da sie Springer- und Vertretungsregelungen vereinfachen, das erholungswirksame Beendigen von Arbeitstätigkeiten unterstützen, Pausenunterbrechungen vorbeugen und Möglichkeiten zur kollektiven Pauseneinnahme fördern (Wendsche und Lohmann-Haislah 2018).

\section{Schlussfolgerungen}

Unsere Studie zeigt, dass Kranken- und Altenpflegekräfte im Vergleich zu anderen Berufsgruppen überhäufig den Ausfall gesetzlich verpflichtender Ruhepausen berichten, vor allem aufgrund arbeitsbedingter und weniger persönlicher Gründe. Der Pausenausfall birgt dabei ein gesteigertes Risiko für berichtete körperliche und psychosomatische Beschwerden in sich. Als zentrale Einflussgrößen für den Ausfall von Ruhepausen und damit Ansatzpunkte für Präventivmaßnahmen identifizierten wir Führungsverantwortung, hohe quantitative Arbeitsanforderungen und atypische Arbeitszeitformen, die mit steigender Arbeitsbelastung einhergehen. Da aufgrund des demografischen Wandels der Pflegebedarf in Deutschland weiter steigen wird, ist es umso wichtiger, Pflegekräfte möglichst lange gesund in ihrem Beruf zu halten. Jenseits der Optimierung von Arbeitstätigkeits-, Arbeitsorganisations- und Arbeitszeitmerkmalen entsprechend ergonomischer Standards, kommt dabei der Förderung solcher Arbeitsressourcen, die beeinträchtigenden Beanspruchungsfolgen zusätzlich vorbeugen können, eine immer wichtigere Rolle zu. Insofern bedarf es zukünftig vermehrter Anstrengungen, um die Einhaltung gesetzlich verpflichtender Ruhepausen bei Pflegekräften in der Praxis besser umzusetzen.

Open Access Dieser Artikel wird unter der Creative Commons Namensnennung 4.0 International Lizenz veröffentlicht, welche die Nutzung, Vervielfältigung, Bearbeitung, Verbreitung und Wiedergabe in jeglichem Medium und Format erlaubt, sofern Sie den/die ursprünglichen Autor(en) und die Quelle ordnungsgemäß nennen, einen Link zur Creative Commons Lizenz beifügen und angeben, ob Änderungen vorgenommen wurden.

Die in diesem Artikel enthaltenen Bilder und sonstiges Drittmaterial unterliegen ebenfalls der genannten Creative Commons Lizenz, sofern sich aus der Abbildungslegende nichts anderes ergibt. Sofern das betreffende Material nicht unter der genannten Creative Commons Lizenz steht und die betreffende Handlung nicht nach gesetzlichen Vorschriften erlaubt ist, ist für die oben aufgeführten Weiterverwendungen des Materials die Einwilligung des jeweiligen Rechteinhabers einzuholen.

Weitere Details zur Lizenz entnehmen Sie bitte der Lizenzinformation auf http://creativecommons.org/licenses/by/4.0/deed.de.

\section{Literatur}

Afentakis A, Maier T (2010) Projektionen des Personalbedarfs und -angebots in Pflegeberufen bis 2025. DeStatis, Wiesbaden

Amlinger-Chatterjee M (2016) Psychische Gesundheit in der Arbeitswelt: atypische Arbeitszeiten. Bundesanstalt für Arbeitsschutz und Arbeitsmedizin, Dortmund

Blau PM (1964) Exchange and power in social life. John Wiley, New York

Bundesagentur für Arbeit (2011) Systematischer und alphabetischer Teil mit Erläuterungen. Klassifikation der Berufe 2010, Bd. 1. Bundesagentur für Arbeit, Nürnberg

Bundesagentur für Arbeit (2018) Statistik/Arbeitsmarktberichterstattung, Berichte: Blickpunkt Arbeitsmarkt - Fachkräfteengpassanalyse. Bundesagentur für Arbeit, Nürnberg

Bundesanstalt für Arbeitsschutz und Arbeitsmedizin (2014) Arbeit in der Pflege - Arbeit am Limit? Arbeitsbedingungen in der Pflegebranche. BIBB/BAuA-Faktenblatt, Bd. 10. Bundesanstalt für Arbeitsschutz und Arbeitsmedizin, Dortmund

Cavanaugh MA, Boswell WR, Roehling MV, Boudreau JW (2000) An empirical examination of self-reported work stress among US managers. J Appl Psychol 85:65-74

Demerouti E, Nachreiner F (2018) Zum Arbeitsanforderungen-Arbeitsressourcen-Modell von Burnout und Arbeitsengagement - Stand der Forschung. Z Arb Wiss. https://doi.org/10.1007/ s41449-018-0100-4

Diehl E, Rieger S, Letzel S, Nienhaus A, Escobar Pinzon LC (2018) Arbeitssituation von Pflegekräften in der spezialisierten Palliativversorgung in Rheinland-Pfalz. ASU Arbeitsmed Sozialmed Umweltmed 53:41-46

DIN N EN ISO 10075-1 (2018) Allgemeine Aspekte und Konzepte und Begriffe. Ergonomische Grundlagen bezüglich psychischer Arbeitsbelastung, Bd. 1. Beuth, Berlin

Fischer D, Lombardi DA, Folkard S, Willetts J, Christiani DC (2017) Updating the "Risk Index": A systematic review and meta-analysis of occupational injuries and work schedule characteristics. Chronobiol Int 34:1423-1438

Gehrke C: Helios muss 88.000 Euro Strafe zahlen. In Berliner Zeitung vom 22./23. April 2017

Glaser J, Höge T (2005) Probleme und Lösungen in der Pflege aus Sicht der Gesundheitswissenschaften. Bundesanstalt für Arbeitsschutz und Arbeitsmedizin, Dortmund, Berlin, Dresden

Gouldner AW (1960) The norm of reciprocity: a preliminary statement. Am Sociol Rev 25:161-178 
Graf O, Rutenfranz J, Ulich E (1970) Arbeitszeit und Arbeitspausen. In: Mayer A, Herwig B (Hrsg) Betriebspsychologie. Handbuch der Psychologie, Bd. 9. Hogrefe, Göttingen, S 244-277

Hasselhorn H-M, Müller BH, Tackenberg P, Kümmerling A, Simon M (2005) Berufsausstieg bei Pflegepersonal: Arbeitsbedingungen und beabsichtigter Berufsausstieg bei Pflegepersonal in Deutschland und Europa. Bundesanstalt für Arbeitsschutz und Arbeitsmedizin, Dortmund, Berlin, Dresden

Ishig A, Hacker W (1999) Arbeits- und Beanspruchungssituation von Pflegepersonal. Vergleichende Untersuchung in Krankenhäusern. Arbeit 8:210-215

Katz D, Kahn RL (1978) The social psychology of organizations, 2. Aufl. Wiley, New York

Keller J, Bless H, Blomann F, Kleinböhl D (2011) Physiological aspects of flow experiences: Skills-demand-compatibility effects on heart rate variability and salivary cortisol. J Exp Soc Psychol 47:849-852

Kliner K, Rennert D, Richter M (Hrsg) (2017) Gesundheit und Arbeit. Blickpunkt Gesundheitswesen. BKK Gesundheitsatlas 2017. Medizinisch Wissenschaftliche Verlagsgesellschaft, Berlin

Krause A, Schwendener S, Berset M, Knecht M, Bogusch K (2017) Team strategies for coping with time pressure. Psychol Everyday Activity 10:4-18

LePine JA, Podsakoff NP, LePine MA (2005) A meta-analytic test of the challenge stressor-hindrance stressor framework: an explanation for inconsistent relationships among stressors and performance. Acad Manage J 48:764-775

Lohmann-Haislah A, Bodnar L (2014) Vom Wert der Pause. ASU Prot $1: 28-33$

Meijman TF, Mulder G (1998) Psychological aspects of workload. In: Drenth PJD, Thierry H, de Wolff CJ (Hrsg) Handbook of work and organizational psychology, 2. Aufl. Bd. 2. Psychology Press, Hove, S 5-33

Nakamura J, Csikszentmihalyi M (2014) The concept of flow. In: Csikszentmihalyi M (Hrsg) Flow and the foundations of positive psychology. Springer, Chicago, S 239-263

Podsakoff NP, LePine JA, LePine MA (2007) Differential challenge stressor-hindrance stressor relationships with job attitudes, turnover intentions, turnover, and withdrawal behavior: a meta-analysis. J Appl Psychol 92:438-454

Rau R, Göllner C (2018) Rahmenmodell der Arbeitsintensität als objektiv bestehende Anforderung. Arbeit 27:151-174

Rohrbach-Schmidt D, Hall A (2013) BIBB/BAuA-Erwerbstätigenbefragung 2012. BIBB-FDZ Daten- und Methodenberichte, Bd. $1 / 2013$. Bundesinstitut für Berufsbildung, Bonn

Rothgang H, Müller R, Unger R (2012) Themenreport „Pflege 2030““. Bertelsmann, Gütersloh

Saloniemi A, Virtanen P, Vahtera J (2004) The work environment in fixed-term jobs: are poor psychosocial conditions inevitable? Work Employ Soc 18:193-208

Schulz-Dadaczynski A (2017) Umgang mit Zeit- und Leistungsdruck: eher Anpassung als Reduktion? Präv Gesundheitsf 12:160-166
Sonnentag S, Venz L, Casper A (2017) Advances in recovery research: What have we learned? What should be done next? J Occup Health Psychol 22:365-380

Statistisches Bundesamt (2017) Pflegestatistik 2015. Pflege im Rahmen der Pflegeversicherung Deutschlandergebnisse. DeStatis, Wiesbaden

Stefancyk AL (2009) One-hour, off-unit meal breaks. Am J Nurs 109:64-66

Tajfel H, Turner JC (1986) The social identity theory of intergroup behavior. In: Worchel S, Austin WG (Hrsg) Psychology of intergroup relations. Nelson Hall, Chicago, S 7-24

Tanner G, Bamberg E, Kersten M, Kozak A, Nienhaus A (2017) The relationship between working time and ill health: differences between full-time and part-time workers. Z Arbeits Organisationspsychol 61:181-196

Theobald H (2018) Pflegearbeit in Deutschland, Japan und Schweden. Hans-Böckler-Stiftung, Düsseldorf

Vereinigung der Bayerischen Wirtschaft e V (2012) Pflegelandschaft 2030. vbw, München

Wendsche J, Lohmann-Haislah A (2017a) A meta-analysis on antecedents and outcomes of detachment from work. Front Psychol $7: 2072$

Wendsche J, Lohmann-Haislah A (2017b) Detachment als Bindeglied zwischen psychi-schen Arbeitsanforderungen und ermüdungsrelevanten psychischen Beanspruchungsfolgen: eine Metaanalyse. Z Arb Wiss 71:52-70

Wendsche J, Lohmann-Haislah A (2018) Arbeitspausen gesundheitsund leistungsförderlich gestalten. Hogrefe, Göttingen

Wendsche J, Ghadiri A, Bengsch A, Wegge J (2017) Antecedents and outcomes of nurses' rest break organization: a scoping review. Int J Nurs Stud 75:65-80

Wendsche J, Hacker W, Wegge J, Schrod N, Roitzsch K, Tomaschek A, Kliegel M (2014) Rest break organization in geriatric care and turnover: A multimethod cross-sectional study. Int J Nurs Stud 51:1246-1257

Wendsche J, Lohmann-Haislah A, Wegge J (2016) The impact of supplementary short rest breaks on task performance-a meta-analysis. SozialpolitikCH 2:1-24

Wilkin CL, de Jong JP, Rubino C (2018) Teaming up with temps: the impact of temporary workers on team social networks and effectiveness. Eur J Work Organ Psychol 27:204-218

Wittig P, Nöllenheidt C, Brenscheidt S (2013) Grundauswertung der BIBB/BAuA-Erwerbstätigenbefragung 2012 mit den Schwerpunkten Arbeitsbedingungen, Arbeitsbelastungen und gesundheitliche Beschwerden. Bundesanstalt für Arbeitsschutz und Arbeitsmedizin, Dortmund

Wöhrmann AM, Gerstenberg S, Hünefeld L, Pundt F, Reeske-Behrens A, Brenscheidt F, Beermann B (2016) Arbeitszeitreport Deutschland 2016. Bundesanstalt für Arbeitsschutz und Arbeitsmedizin, Dortmund 